\title{
MONODROMIES OF HYPERELLIPTIC FAMILIES OF GENUS THREE CURVES
}

\author{
MizUHo IsHIZAKA
}

(Received May 15, 2001, revised October 21, 2003)

\begin{abstract}
A complete list of the monodromies of degenerations of genus three which are not realized as the monodromies of any hyperelliptic families of genus three is given. We also prove that all the other monodromies of genus three are realized as the monodromies of certain hyperelliptic families.
\end{abstract}

Introduction. Let $\phi: S \rightarrow \Delta$ be a proper surjective holomorphic map from a nonsingular complex surface $S$ to a small disk $\Delta:=\{t \in C|| t \mid<\varepsilon\}$ such that $\phi^{-1}(t)$ is a nonsingular curve of genus $g \geq 2$ for each $t \in \Delta^{*}:=\Delta \backslash\{0\}$. We call $(\phi, S, \Delta)$ a degeneration of curves of genus $g$. If all $\phi^{-1}(t)$ for $t \in \Delta^{*}$ are hyperelliptic curves, we call $(\phi, S, \Delta)$ a hyperelliptic family. We set $X:=\phi^{-1}(0)$ and call it the special fiber of $S$. If the reduced scheme of $X$ has normal crossings as singularities and any $(-1)$-curve in the special fiber intersects the other components at at least three points, $(\phi, S, \Delta)$ is said to be normally minimal. Two degenerations $(\phi, S, \Delta)$ and $\left(\phi^{\prime}, S^{\prime}, \Delta^{\prime}\right)$ are said to be topologically equivalent if there exist orientation-preserving homeomorphisms $\psi: S \rightarrow S^{\prime}$ and $\bar{\psi}: \Delta \rightarrow \Delta^{\prime}$ satisfying $\phi^{\prime} \circ \psi=\bar{\psi} \circ \phi$.

Let $\mathcal{T}_{g}:=$ nnormally minimal degenerations of genus $\left.g\right\} / \sim$, where $\sim$ is the topological equivalence. For an element of $\mathcal{T}_{g}$, we can uniquely determine the topological monodromy (sometimes called the monodromy, for short) as a conjugacy class in the mapping class group of genus $g$. The monodromy of a degeneration is a conjugacy class of a pseudo-periodic map of negative type (cf. [MM1], [Ni1], [Ni2], [Im], [ES], [ST], [AMO] etc.). Conversely, any conjugacy class of a pseudo-periodic map of negative type is realized as the monodromy of a certain degeneration (cf. [MM2]). In [AI], using the theory of Harvey and Wiman (cf. [Ha], [Wi]) and the list of the stable curves of genus three in [F], we classified the monodromies of degenerations of curves of genus three together with their topological types of moduli points.

In this paper, we completely classify the monodromies of degenerations of genus three that cannot be realized as the monodromies of any hyperelliptic families of genus three (Theorem 1.8). Moreover, we prove that all the other monodromies of genus three are realized as the monodromies of certain hyperelliptic families. For the classification, we define an operation called the "inverse of Horikawa's canonical resolution". Using this operation, we easily see that the closure of the hyperelliptic locus $\overline{\boldsymbol{H}_{3}}$ in the Deligne-Mumford compactification $\overline{M_{3}}$ of the moduli space of genus three curves does not intersect the strata of the stable curves

2000 Mathematics Subject Classification. Primary 14D06; Secondary 14H45, 14H15, 57M99, 30 F99. 
of types (D), (H), (I), (M), (O) (cf. Corollary 1.6). In order to prove the existence of hyperelliptic families with monodromies not listed in Theorem 1.8, we give, for each monodromy, the defining equation of a hyperelliptic family with the monodromy.

When we deal with the monodromies of hyperelliptic families, we need to deal carefully with the data of monodromies called the screw number.

The conjugacy class of a pseudo-periodic map $f: \Sigma_{g} \rightarrow \Sigma_{g}$ of negative type of genus $g$ (i.e., the monodromy of a degeneration) can be determined by the following data (cf. [MM2]): (i) an admissible sysytem of cut curves $\mathcal{C}=\amalg C_{i}$ on $\Sigma_{g}$, (ii) an action of $f$ on the oriented graph induced by the admissible system, (iii) the valency data of the stabilizer of each component of $\Sigma \backslash \mathcal{C}$, (iv) the screw number of $f$ around each neighborhood of $C_{i}$.

For example, there exist conjugacy classes $\left[f_{1}\right]$ and $\left[f_{2}\right]$ of pseudo-periodic maps of negative type such that $\left[f_{1}\right]$ can be realized as the monodromy of a hyperelliptic family but not $\left[f_{2}\right]$, although their data (i), (ii) and (iii) coincide.

In Section 1, we first classify the monodromies among those listed in [AI] that cannot be realized as monodromies of any hyperelliptic family of genus three (cf. the list in Theorem 1.8). More precisely, for each monodromy $[f]$ listed in Theorem 1.8, we prove that any family whose monodromy is $[f]$ cannot be obtained by Horikawa's canonical resolution of any double covering of $\boldsymbol{P}^{1} \times \Delta$. We also show in Theorem 1.8 that all monodromies in [AI] not listed in Theorem 1.8 can be realized as the monodromies of certain hyperelliptic families. In Section 2, we prove this by constructing families whose monodromies are not listed in Theorem 1.8 .

In this paper, We adopt the same terminology for topological monodromies of genus three as in [AI].

The author thanks Professors Tadashi Ashikaga, Kazuhiro Konno, Masanori Ishida, Tatsuya Arakawa, Takeshi Kajiwara and Shigeru Takamura, for their useful advice and valuable discussions. He wishes to express his special gratitude to Professor Tadao Oda for his continuous encouragement.

\section{Possibility for the existence.}

1.1. Inverse of canonical resolution. We first review Horikawa's canonical resolution of singularities appearing in double coverings of a surface (cf. [Ho1, §2]). Let $\phi: S \rightarrow \Delta$ be a normally minimal hyperelliptic family of genus $g$. By the same argument as in [Ho2, §1], we see that $S$ is bimeromorphic to a double covering $\psi_{0}: S_{0} \rightarrow W_{0}:=\boldsymbol{P}^{1} \times \Delta$ branched along a divisor $B_{0}$ of $W_{0}$. More precisely, there exists a line bundle $F_{0}$ on $\boldsymbol{P}^{1} \times \Delta$ such that the line bundle $\left[B_{0}\right]$ associated to $B_{0}$ is isomorphic to $F_{0}^{\otimes 2}$ and that $S_{0}$ is realized in the total space of $F_{0}$ as a double covering of $\boldsymbol{P}^{1} \times \Delta$. Let $\pi_{0}$ be the second projection of $W_{0}$. We set $\Gamma_{t}:=\pi_{0}^{-1}(t), \tilde{B}_{0}:=B_{0}-\Gamma_{0}$ when $\Gamma_{0}$ is a component of $B_{0}$, and $\tilde{B}_{0}:=B_{0}$ otherwise. The pair $\left(S_{0}, B_{0}\right)$ satisfies the following conditions:

(i) The intersection number $B_{0} \cdot \Gamma_{t}$ is equal to $2 g+2$.

(ii) If the local intersection number $I_{P}\left(\tilde{B}_{0}, \pi_{0}^{-1}(t)\right)$ of $\tilde{B}_{0}$ and $\Gamma_{t}$ at $P$ is greater than one, then $P$ is on $\Gamma_{0}$. 
We define $\tau_{i}, \tilde{\tau}_{i}, \pi_{i}, B_{i}, \tilde{B}_{i}, F_{i}, \tilde{\Gamma}_{i}, E_{i}$ and $\psi_{i}$ inductively as follows: Let $\tau_{0}$ be the identity map of $W_{0}$. We choose a bad point $P_{i-1}$ on $B_{i-1}$, that is, $P_{i-1}$ is a singular point or satisfies $I_{P_{i-1}}\left(\tilde{B}_{i-1},\left(\left(\tau_{0} \circ \cdots \circ \tau_{i-1}\right)^{*} \Gamma_{0}\right)_{\text {red }}\right) \geq 2$, where $\tilde{B}_{i-1}$ is the strict transform of $\tilde{B}_{0}$ by $\tau_{0} \circ \cdots \circ \tau_{i-1}$. Let $\tau_{i}: W_{i} \rightarrow W_{i-1}$ be the blowing-up at $P_{i-1}$. We denote the multiplicity of $B_{i-1}$ at $P_{i-1}$ by $m_{P_{i-1}}$. Let $E_{i}$ be the exceptional set of $\tau_{i}$. We define $\tilde{\Gamma}_{i-1}$ as the reduced scheme of $\left(\tau_{0} \circ \cdots \circ \tau_{i-1}\right)^{*} \Gamma_{0}$. We set $B_{i}:=\tau_{i}^{*} B_{i-1}-2\left[m_{P_{i-1}} / 2\right] E_{i}$ and $F_{i}:=\tau_{i}^{*} F_{i-1}-\left[m_{P_{i-1}} / 2\right] E_{i}$, where $\left[m_{P_{i-1}} / 2\right]$ is the greatest integer not exceeding $m_{P_{i-1}} / 2$. Since $\left[B_{i}\right] \simeq F_{i}^{\otimes 2}$, we can take a double covering $\psi_{i}: S_{i} \rightarrow W_{i}$ branched along $B_{i}$ in the total space of $F_{i}$, and naturally define a bimeromorphic map $\tilde{\tau}_{i}: S_{i} \rightarrow S_{i-1}$ (cf. [Ho1, §2]). We set $\pi_{i}:=\pi_{i-1} \circ \tau_{i}$. Repeating this process at all bad points, we obtain a sequence of blowing-ups $W_{r} \stackrel{\tau_{r}}{\rightarrow} \cdots \rightarrow W_{1} \stackrel{\tau_{1}}{\rightarrow} W_{0}$ satisfying the following properties:

(a) $B_{r}$ is nonsingular.

(b) $\Theta:=\left(\tau_{1} \circ \cdots \circ \tau_{r}\right)^{*}\left(\Gamma_{0}\right)$ and the strict transform of $\tilde{B}_{0}$ intersect each other transversally.

$S_{r}$ is nonsingular by (a). The reduced scheme of the special fiber of $S_{r}$ is a normal crossing divisor by (b). We obtain the original normally minimal model $\phi: S \rightarrow \Delta$ by the composite of the blowing-downs of suitable $(-1)$-curves successively on $S_{r}$. We call the above process Horikawa's canonical resolution (the canonical resolution, for short). In this paper, we always use $r$ as the length of the sequence of the blowing-ups that satisfies the conditions (a) and (b).

Conversely, choosing a component $E_{r}^{\prime}$ of $\left(\tau_{1} \circ \cdots \circ \tau_{r}\right)^{*}\left(\Gamma_{0}\right)$ whose self-intersection number is -1 , we consider the blowing-down $\tau_{r}^{\prime}: W_{r} \rightarrow W_{r-1}^{\prime}$ which contracts $E_{r}^{\prime}$ to a point $P^{\prime}$. We set $B_{r}^{\prime}:=B_{r}-E_{r}^{\prime}$ when $E_{r}^{\prime}$ is a component of $B_{r}$, and $B_{r}^{\prime}:=B_{r}$ otherwise. Let $m_{P^{\prime}}$ be the intersection number $E_{r}^{\prime} \cdot B_{r}^{\prime}$. Since $\left(\tau_{r}^{\prime}\right)_{*}\left(B_{r}+2\left[m_{P^{\prime}} / 2\right] E_{r}^{\prime}\right)$ is isomorphic to $\left(\tau_{r}^{\prime}\right)_{*}\left(F_{r}+\left[m_{P^{\prime}} / 2\right] E_{r}^{\prime}\right)^{\otimes 2}$, we can take the double covering $\psi_{r-1}^{\prime}: S_{r-1}^{\prime} \rightarrow W_{r-1}^{\prime}$ branched along $\left(\tau_{r}^{\prime}\right)_{*} B_{r}$ and naturally define a morphism $\tilde{\tau}_{r}^{\prime}: S_{r} \rightarrow S_{r-1}^{\prime}$. Repeating this process, we finally obtain a sequence of blowing-downs $W_{r} \stackrel{\tau_{r}}{\rightarrow} \cdots \rightarrow W_{1}^{\prime} \stackrel{\tau_{1}}{\rightarrow} W_{0}^{\prime}$ and a double covering $\psi_{r}^{\prime}: S_{0}^{\prime} \rightarrow W_{0}^{\prime}=\boldsymbol{P}^{1} \times \Delta$ such that $S_{0}^{\prime}$ is bimeromorphic to $S_{r}$. We call this process an inverse of Horikawa's canonical resolution. Note that if the multiplicity of a component $E$ of $\left(\tau_{1} \circ \cdots \circ \tau_{r}\right)^{*} \Gamma_{0}$ is one, we can find an inverse of Horikawa's canonical resolution such that $\left(\tau_{1}^{\prime} \circ \cdots \circ \tau_{r}^{\prime}\right)_{*} E$ is $\boldsymbol{P}^{1}$, i.e., we can consider $\left(\tau_{1}^{\prime} \circ \cdots \circ \tau_{r}^{\prime}\right)_{*} E$ to be $\Gamma_{0}$. We call this an inverse of Horikawa's canonical resolution associated to $E$. Let $C$ be a prime divisor of $S$ that is a component of $\phi^{-1}(0)$. Let $Z$ be the set of points that are the images of the exceptional curves of $\tilde{\tau}: S_{r} \rightarrow S$. Let $\Pi(C):=\overline{\psi_{r} \circ \tilde{\tau}^{-1}(C-(C \cap Z))}$ denote the closure of $\psi_{r} \circ \tilde{\tau}^{-1}(C-(C \cap Z))$ in $W_{r} . \Pi(C)$ is also a prime divisor on $W_{r}$. Assume that $C^{\prime}$ is another component of $\phi^{-1}(0)$ satisfying $\Pi(C) \cap \Pi\left(C^{\prime}\right)=\emptyset$. Since the dual graph of $\Theta$ is connected, there exists a subdivisor $D_{C C^{\prime}}=\sum a_{i} E_{i}$ of $\Theta$ that satisfies the following conditions (we use the same symbol $E_{i}$ for the strict transform of $E_{i}$ on $W_{r}$ ):

(i) $\Theta \geq D_{C C^{\prime}}$ and $\Theta \nsucceq D_{C C^{\prime}}+E_{i}$ for all $E_{i}\left(a_{i} \neq 0\right)$.

(ii) $\operatorname{Supp}(\Pi(C)) \cap D_{C C^{\prime}} \neq \emptyset$ and $\operatorname{Supp}\left(\Pi\left(C^{\prime}\right)\right) \cap D_{C C^{\prime}} \neq \emptyset$.

(iii) $D_{C C^{\prime}} \geq \Pi(C)$ and $D_{C C^{\prime}} \geq \Pi\left(C^{\prime}\right)$. 
(iv) $\operatorname{Supp}\left(D_{C C^{\prime}}\right)$ is connected.

Since the dual graph of $\Theta$ has no loop, $D_{C C^{\prime}}$ is uniquely determined. We set $D_{C C^{\prime}}=0$ when $\Pi(\mathrm{C})$ intersects $\Pi\left(\mathrm{C}^{\prime}\right)$. We call the divisor $D_{C C^{\prime}}$ the bridge between $\Pi(C)$ and $\Pi\left(C^{\prime}\right)$.

1.2. Periodic case. Let $\Delta^{\prime} \rightarrow \Delta$ be a totally ramified cover of degree $d$ branched at the origin. Let $S_{d}$ be the nonsiglular model of $S \times \Delta \Delta^{\prime}$ and $\phi_{d}: S_{d} \rightarrow \Delta^{\prime}$ the natural morphism. Let $f$ be a representative of the monodromy of $(\phi, S, \Delta)$ (namely, $f$ is a pseudoperiodic map and its conjugacy class $[f]$ in the mapping class group is the monodromy of $(\phi, S, \Delta))$. Then the monodromy of $\left(\phi_{d}, S_{d}, \Delta^{\prime}\right)$ is [ $\left.f^{d}\right]$. In Lemma 1.4 of [AI], we classified the conjugacy classes of periodic maps of genus $g(1 \leq g \leq 3)$. The data for the conjugacy class of a periodic map $[f]$ consists of two invariants: the period and the total valency. The period $n$ is the smallest positive integer such that $f^{n}$ is isotopic to the identity.

We introduce the notion of the valency originally defined by Nielsen ([Ni1]). By Kerchhoff's theorem (cf. [Ke]), for each periodic homeomorphism $f$, there exist a Riemann surface $\Sigma_{g}$ of genus $g$ and an analytic automorphism $\bar{f}: \Sigma_{g} \rightarrow \Sigma_{g}$ isotopic to $f$. For each point $P$ on $\Sigma_{g}$, we denote by $r_{P}$ the cardinality of the orbit of $P$ under $\bar{f}$, and let $l_{P}:=n / r_{P}$. Let $\delta_{P}$ be the smallest nonnegative integer such that $\bar{f}^{r_{P}}$ is the rotation of angle $2 \pi \delta_{P} / l_{P}$ near each point in the orbit. Denote by $s_{P}$ the smallest positive integer satisfying $\delta_{P} s_{P} \equiv 1\left(\bmod l_{P}\right)$ if $\delta_{P} \neq 0$, and set $s_{P}:=0$ when $\delta_{P}=0$. The symbol $s_{P} / l_{P}$ is called the valency of the orbit of $P$.

Note that the valencies of all but a finite number of orbits are zero. The set of the positive valencies is called the total valency of $\bar{f}$ and expressed as the formal sum $\sum s_{P} / l_{P}$ of symbols.

We define the total valency of a periodic homeomorphism $f$ as the total valency of $\bar{f}$. It is well-known that the conjugacy class of a periodic map is determined by its period and total valency.

For instance, (1) in [AI, p. 202], $n=14 ; 11 / 14+5 / 7+1 / 2$ means that there exist three orbits $\mathcal{O}_{1}=\left\{P_{1}\right\}, \mathcal{O}_{2}=\left\{P_{2}, \bar{f}^{2}\left(P_{2}\right)\right\}$, and $\mathcal{O}_{3}=\left\{P_{3}, \bar{f}^{2}\left(P_{3}\right), \cdots, \bar{f}^{6}\left(P_{3}\right)\right\}$ such that $\bar{f}$ is the rotaion of angle $2 \pi \times 9 / 14$ near $P_{1}, \bar{f}^{2}$ is the rotaion of angle $2 \pi \times 3 / 7$ near each point in $\mathcal{O}_{2}$ and $\bar{f}^{7}$ is the rotaion of angle $\pi$ near each point in $\mathcal{O}_{3}$.

We use the same symbols as in [AI, p. 202 and p. 203]. To avoid confusion with another number in another paragraph in [AI, p. 203], we denote for example by (i1) the monodromy (1) in [AI, p. 202], $n=14 ; 11 / 14+5 / 7+1 / 2$.

Let $S$ be a family of genus three with periodic monodromy (i1) and $\bar{f}$ as above. Taking a base change of degree seven, we obtain a family $S_{7} \rightarrow \Delta$ whose monodromy is a periodic map with $n=2 ; 1 / 2+1 / 2+1 / 2+1 / 2+1 / 2+1 / 2+1 / 2+1 / 2$, because the points in $\mathcal{O}_{1}$ and $\mathcal{O}_{3}$ are the fixed points of the involution $\bar{f}^{7}$. Repeating this calculation for all periodic maps of genus three, we obtain the following:

LEMMA 1.1. By taking a base change of suitable degree, all periodic maps of genus three are obtained from those with

(i1) $n=14,11 / 14+5 / 7+1 / 2$, 
(i7) $n=12,11 / 12+7 / 12+1 / 2$,

(i9) $n=12,11 / 12+3 / 4+1 / 3$,

(i13) $n=9,8 / 9+4 / 9+2 / 3$,

(i20) $n=8,1 / 8+5 / 8+1 / 4$,

(i22) $n=8,3 / 8+3 / 8+1 / 4$,

(i28) $n=7,1 / 7+2 / 7+4 / 7$,

(i44) $n=4, g^{\prime}=1,1 / 2+1 / 2$,

(i47) $n=2, g^{\prime}=2$ and $\Pi: \Sigma_{g} \rightarrow \Sigma_{g^{\prime}}$ is an unramified covering.

Proof. We set $m_{1}=$ (i1), $m_{7}:=$ (i7), $m_{9}:=$ (i9), $m_{13}:=$ (i13), $m_{20}:=$ (i20), $m_{22}:=(\mathrm{i} 22), m_{28}:=$ (i28). Then, by elementary calculations, we obtain the following equations:

$$
\begin{array}{llll}
\left(m_{1}\right)^{2}=(\mathrm{i} 31), & \left(m_{1}\right)^{3}=(\mathrm{i} 3), & \left(m_{1}\right)^{4}=(\mathrm{i} 25), & \left(m_{1}\right)^{5}=(\mathrm{i} 6), \\
\left(m_{1}\right)^{6}=(\mathrm{i} 29), & \left(m_{1}\right)^{7}=(\mathrm{i} 43), & \left(m_{1}\right)^{8}=(\mathrm{i} 30), & \left(m_{1}\right)^{9}=(\mathrm{i} 5), \\
\left(m_{1}\right)^{10}=(\mathrm{i} 26), & \left(m_{1}\right)^{11}=(\mathrm{i} 4), & \left(m_{1}\right)^{12}=(\mathrm{i} 32), & \left(m_{1}\right)^{13}=(\mathrm{i} 2), \\
\left(m_{7}\right)^{2}=(\mathrm{i} 33), & \left(m_{7}\right)^{3}=(\mathrm{i} 39), & \left(m_{7}\right)^{4}=(\mathrm{i} 45), & \left(m_{7}\right)^{7}=(\mathrm{i} 8), \\
\left(m_{7}\right)^{9}=(\mathrm{i} 40), & \left(m_{9}\right)^{2}=(\mathrm{i} 34), & \left(m_{9}\right)^{3}=(\mathrm{i} 36), & \left(m_{9}\right)^{4}=(\mathrm{i} 42), \\
\left(m_{9}\right)^{5}=(\mathrm{i} 11), & \left(m_{9}\right)^{6}=(\mathrm{i} 46), & \left(m_{9}\right)^{7}=(\mathrm{i} 12), & \left(m_{9}\right)^{8}=(\mathrm{i} 41), \\
\left(m_{9}\right)^{10}=(\mathrm{i} 35), & \left(m_{9}\right)^{11}=(\mathrm{i} 10), & \left(m_{13}\right)^{2}=(\mathrm{i} 16), & \left(m_{13}\right)^{4}=(\mathrm{i} 18), \\
\left(m_{13}\right)^{5}=(\mathrm{i} 17), & \left(m_{13}\right)^{6}=(\mathrm{i} 42), & \left(m_{13}\right)^{7}=(\mathrm{i} 15), & \left(m_{13}\right)^{8}=(\mathrm{i} 14), \\
\left(m_{20}\right)^{2}=(\mathrm{i} 37), & \left(m_{20}\right)^{3}=(\mathrm{i} 19), & \left(m_{22}\right)^{3}=(\mathrm{i} 24), & \left(m_{22}\right)^{6}=(\mathrm{i} 38), \\
\left(m_{22}\right)^{5}=(\mathrm{i} 23), & \left(m_{22}\right)^{7}=(\mathrm{i} 21), & \left(m_{28}\right)^{3}=(\mathrm{i} 27) . &
\end{array}
$$

LEMMA 1.2. Let $E$ be a component of $\left(\tau_{1} \circ \cdots \circ \tau_{i}\right)^{*}\left(\Gamma_{0}\right)$ whose multiplicity $\alpha$ is greater than or equal to two. Assume that $E$ intersects at least three distinct components $E_{j_{1}}$, $E_{j_{2}}, E_{j_{3}}$ of $\left(\tau_{1} \circ \cdots \circ \tau_{i}\right)^{*}\left(\Gamma_{0}\right)$. Let $\hat{E}_{j_{i}}(i=1,2,3)$ be mutually distinct maximal connected subdivisors of $\left(\tau_{1} \circ \cdots \circ \tau_{i}\right)^{*}\left(\Gamma_{0}\right)$ such that their supports do not contain $E$ and that $\hat{E}_{j_{i}} \geq E_{j_{i}}$. In any inverse of the canonical resolution, at least one of the $\hat{E}_{j_{i}}(i=1,2,3)$ is contracted before $E$.

PROOF. Let $\tau_{r^{\prime}}$ be the blowing-up such that the strict transform of $E_{r^{\prime}}$ by $\tau_{r^{\prime}+1} \circ \cdots \circ \tau_{i}$ is $E$. If none of the $\hat{E}_{j_{i}}(i=1,2,3)$ are contracted before $E$ in any inverse of the canonical resolution, three distinct nonzero subdivisors $\left(\tau_{r^{\prime}} \circ \cdots \circ \tau_{i}\right)\left(\hat{E}_{j_{i}}\right)(i=1,2,3)$ intersect at a point on $W_{r^{\prime}-1}$. However, considering the process of the canonical resolutions, we see that the singularities of the reduced scheme of $\left(\tau_{1} \circ \cdots \circ \tau_{s}\right)^{*}\left(\Gamma_{0}\right)$ are ordinary double points for all $s$, a contradiction.

COROLLARY 1.3. In the notation as above, there exists a subdivisor $D$ of $\left(\tau_{1} \circ \cdots \circ \tau_{i}\right)^{*}\left(\Gamma_{0}\right)$ such that one of $\hat{E}_{j_{1}}, \hat{E}_{j_{2}}, \hat{E}_{j_{3}}$ coincides with $\alpha D$.

PROPOSITION 1.4. There exist no hyperelliptic families whose topological monodromies are (i9), (i10), (i11), (i12), (i13), (i14), (i15), (i16), (i17), (i18), (i19), (i20), (i28), (i34), (i35), (i36), (i37), (i41), (i42). 
PROOF. By the argument in the proof of Lemma 1.1, we see that by taking a base change of suitable degree, the periodic monodromies listed above become one of the following: (i28) $n=7 ; 1 / 7+2 / 7+4 / 7$, (i37) $n=4 ; 1 / 4+1 / 4+1 / 4+1 / 4$, (i42) $n=3$; $1 / 3+1 / 3+1 / 3+1 / 3+2 / 3$. Thus, it suffices to prove that there exist no hyperelliptic families whose monodromies are one of them.

Assume that there exists a hyperelliptic family $S$ whose monodromy is (i28). Let $C_{0}$, $C_{1}, C_{2}, C_{3}$ be the components of the special fiber of $S$ whose multiplicities are 7, 1,2 and 4 , respectively, and $C_{0}$ intersects $C_{1}, C_{2}$ and $C_{3}$. Since their multiplicities are distinct, $\Pi\left(C_{i}\right)$ are all distinct. Since the multiplicity of each $\Pi\left(C_{i}\right)$ is not a multiple of seven and the bridge $D_{C_{0} C_{i}}$ intersects $\Pi\left(C_{0}\right)$ at a point, Lemma 1.2 implies the nonexistence. Case (i42) is similar to Case (i28).

Assume that there exists a hyperelliptic family $S$ whose monodromy is (i37). Let $C_{0}$, $C_{1}, \ldots, C_{4}$ be the components of the special fiber of $S$ whose multiplicities are $4,1, \ldots$, 1 , respectively. If $\Pi\left(C_{0}\right)$ is a component of $B_{r}, \Pi\left(C_{0}\right)$ intersects at least four components. It contradicts Lemma 1.2. Thus we may assume that $\Pi\left(C_{0}\right)$ is not a component of $B_{r}$ with multiplicity four, $\Pi\left(C_{1}\right)=\Pi\left(C_{2}\right)$ and $\Pi\left(C_{3}\right)=\Pi\left(C_{4}\right)$ with multiplicity one, respectively. If the bridge $D_{C_{0} C_{1}}$ intersects $B_{r}$ or contains a component of $B_{r}, \psi_{r}^{*}\left(D_{C_{0} C_{1}}\right)$ is connected. In this case, $\tilde{\tau}\left(\psi_{r}^{*}\left(D_{C_{0} C_{1}}\right)\right)$ is a point, and $C_{0}, C_{1}$ and $C_{2}$ intersect at a point, a contradiction. Thus, if $D_{C_{0} C_{1}} \neq 0$, all components of $D_{C_{0} C_{1}}$ are not components of $B_{r}$ and do not intersect $B_{r}$. Since the multiplicities of the components of $\psi_{r}^{*}\left(D_{C_{0} C_{1}}\right)$ are greater than or equal to five, the multiplicities of the components of $D_{C_{0} C_{1}}$ are greater than or equal to five. Then, by any inverse of the canonical resolution, $D_{C_{0} C_{1}}$ is contracted before $\Pi\left(C_{0}\right)$ and $\Pi\left(C_{1}\right)$ are contracted. It means $D_{C_{0} C_{1}}=0$ because, by our definition of the canonical resolution, we do not blow up at non-bad points. By the same argument, $\Pi\left(C_{0}\right)$ intersects $\Pi\left(C_{3}\right)$ at a point. Thus, there exists no component of $\left(\tau_{1} \circ \cdots \circ \tau_{r}\right)^{*}\left(I_{0}\right)$ whose self-intersection number is -1 , a contradiction.

1.3. Non-periodic case. By the semistable reduction theorem (cf. [DM]), there exists a branched cover $\Delta^{\prime} \rightarrow \Delta$ totally ramified over the origin with degree $d$ such that $S_{d} \rightarrow \Delta^{\prime}$ is a semistable family. We call it a semistable model of $\phi: S \rightarrow \Delta$. Let $S_{d} \rightarrow S^{\prime}$ be a composite of the blowing-downs of $(-2)$-curves so that $S^{\prime}$ is free from $(-2)$-curves. We call $S^{\prime} \rightarrow \Delta^{\prime}$ a stable model of $\phi: S \rightarrow \Delta$. We sometimes call the special fiber of a (semi)stable model of $\phi: S \rightarrow \Delta$ the (semi) stable model of the special fiber of $\phi: S \rightarrow \Delta$. We introduce the weighted graphs (A) through $(\mathrm{O})$ as the dual graphs of the stable curves in Table 1 (cf. Table 2 in [AI]). A vertex $v$ corresponds to a component of a stable curve and an edge corresponds to an intersection of two components. Let $g(v)$ and $\rho(v)$ be the genus and the number of singular points of the component $v$, respectively. The number inside a small circle in Table 1 means $g(v)+\rho(v)$. We omit the number when it is zero. For instance, the graph (B) represents six stable curves, that is, $v_{1}$ has genus $i_{1}$ and $2-i_{1}$ singular points while $v_{2}$ has genus $i_{2}$ and $1-i_{2}$ singular points $\left(0 \leq i_{1} \leq 2,0 \leq i_{2} \leq 1\right)$. We write the stable curves $B_{i_{1} i_{2}}$ $\left(0 \leq i_{1} \leq 2,0 \leq i_{2} \leq 1\right)$ for short. Figures $(\mathrm{A})$ to $(\mathrm{O})$ in Table 1 can be regarded as the 
dual graphs of stable curves of genus three. Furthermore, if we replace each edge of these graphs by a chain of $(-2)$-curves, these graphs can be regarded as the weighted dual graphs of semistable curves of genus three. We call a chain of $(-2)$-curves a $\boldsymbol{P}^{1}$-chain at the edge. The number of components of a $\boldsymbol{P}^{1}$-chain is called the length of the $\boldsymbol{P}^{1}$-chain.

PROPOSITION 1.5. There exists no hyperelliptic family of genus three whose special fiber of the stable model has a topological type of either (D), (H), (I), (M) or (O) in Table 1.

TABLE 1. Stable curves of genus 3.

(A)

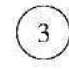

$v_{1}$

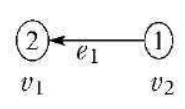

(E)
(B)

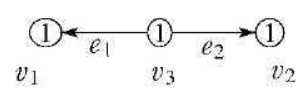

(F)

(C)

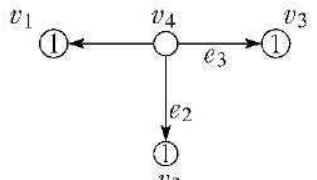

(G) $\quad v$
(1) $\stackrel{e_{1}}{e_{2}}$

(H)

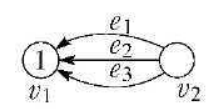

(K)

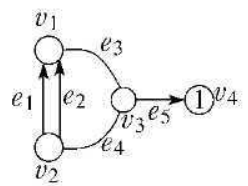

(N)

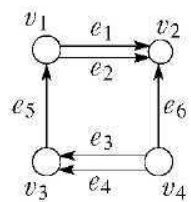

(I)

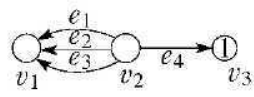

(L)

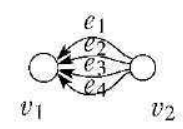

(O) $\underset{v_{1}}{\stackrel{e_{1}}{e_{2}} v_{2}} \stackrel{e_{3}}{\longrightarrow}(1)$

(J)

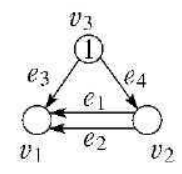

(M)

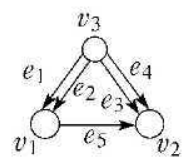

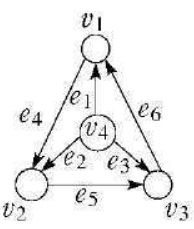


PROOF. Since the degeneration obtained by a base change of a hyperelliptic family is a hyperelliptic family, we may assume the hyperelliptic family $\phi: S \rightarrow \Delta$ is (semi)stable. The vertices of the graphs are regarded as the corresponding irreducible components for the simplicity of notation.

Assume that there exists a hyperelliptic family whose special fiber is (D). Since the multiplicity of each $v_{i}$ is one, $\Pi\left(v_{i}\right)$ is not a component of $B_{r}$. Since $v_{i}(1 \leq i \leq 3)$ intersects $v_{4}$ at a point, the bridge $D_{v_{i} v_{4}}$ between $\Pi\left(v_{i}\right)$ and $\Pi\left(v_{4}\right)$ intersects $v_{4}$ at one point $P_{i}$. Moreover, each $P_{i}$ is contained in $B_{r}$. It means that $v_{4}$ is the double covering of $\boldsymbol{P}^{1}$ branched at least at three points. This contradicts the fact that $v_{4}$ is $\boldsymbol{P}^{1}$.

Assume that there exists a hyperelliptic family whose special fiber is $(\mathrm{H}) . \Pi\left(v_{1}\right)$ does not coincide with $\Pi\left(v_{2}\right)$, because $v_{1}$ is not homeomorphic to $v_{2}$. Since $v_{1}$ is connected with $v_{2}$ by three $\boldsymbol{P}^{1}$-chains, there exist at least two bridges between $\Pi\left(v_{1}\right)$ and $\Pi\left(v_{2}\right)$, a contradiction.

Assume that there exists a hyperelliptic family whose special fiber is (I). Since $v_{3}$ is not homeomorphic to $v_{2}$, we have $\Pi\left(v_{2}\right) \neq \Pi\left(v_{3}\right) . \Pi\left(v_{2}\right)$ intersects $D_{v_{2} v_{3}}$ at a point on $B_{r}$, because $v_{2}$ is connected with $v_{3}$ by a $\boldsymbol{P}^{1}$-chain. Since $\Pi\left(v_{2}\right)$ intersects $B_{r}$, we see that $\Pi\left(v_{1}\right) \neq \Pi\left(v_{2}\right)$. Since $v_{1}$ is connected with $v_{2}$ by three $\boldsymbol{P}^{1}$-chains, there exist at least two bridges between $\Pi\left(v_{1}\right)$ and $\Pi\left(v_{2}\right)$, a contradiction.

Assume that there exists a hyperelliptic family whose special fiber is (M). Since the dual graph of $\Theta$ has no loop, we have $\Pi\left(v_{1}\right)=\Pi\left(v_{2}\right)$. Since $\Pi\left(v_{1}\right) \neq \Pi\left(v_{3}\right)$, there exist at least two bridges between $\Pi\left(v_{3}\right)$ and $\Pi\left(v_{1}\right)$, a contradiction.

Assume that there exist a hyperelliptic family whose special fiber is $(\mathrm{O})$. We may assume $\Pi\left(v_{1}\right)=\Pi\left(v_{2}\right)$ and $\Pi\left(v_{3}\right)=\Pi\left(v_{4}\right)$. In view of the configuration of $(\mathrm{O})$, there exist at least two bridges between $\Pi\left(v_{1}\right)$ and $\Pi\left(v_{3}\right)$, a contradiction.

COROLlary 1.6. Let $\overline{\boldsymbol{M}_{3}}$ be the Deligne-Mumford compactification of the moduli space of curves of genus three. The closure of the hyperelliptic locus $\overline{\boldsymbol{H}_{3}}$ in $\overline{\mathbf{M}_{3}}$ does not intersect the loci of the stable curves whose topological types are (D), (H), (I), (M) and (O).

Proof. Let $C$ be a stable curve whose moduli point is on $\overline{\boldsymbol{H}_{3}}$. According to $[\mathrm{HM}$, Theorem 3.160], there exists an admissible double cover $\pi: C \rightarrow B$ of a stable 8-marked curve $B$ of genus 0 . In the proof of Proposition 1.5, we showed that we cannot construct stable curves of type (D), (H), (I), (M) and (O) as a double cover of genus 0 curves.

PROPOSITION 1.7. There exists no (semi) stable hyperelliptic family whose special fiber of the stable model has a topological type of either $(\mathrm{E}),(\mathrm{F}),(\mathrm{G}),(\mathrm{J}),(\mathrm{K})$ or $(\mathrm{N})$, if the following conditions are satisfied:

(i) The length of the $\boldsymbol{P}^{1}$-chains at $e_{1}$ and $e_{2}$ are mutually distinct in the cases $(\mathrm{E}),(\mathrm{F})$ and $(\mathrm{G})$.

(ii) The length of the $\boldsymbol{P}^{1}$-chains at $e_{3}$ and $e_{4}$ are mutually distinct in the cases $(\mathrm{J})$ and (K).

(iii) The length of the $\boldsymbol{P}^{1}$-chains at $e_{5}$ and $e_{6}$ are mutually distinct in the case $(\mathrm{N})$. 
PROOF. If there exists a family whose special fiber is (E) or (G) satisfying the above condition (i), then we see that $\Pi\left(v_{1}\right) \neq \Pi\left(v_{2}\right)$ and there exist at least two bridges between $\Pi\left(v_{1}\right)$ and $\Pi\left(v_{2}\right)$, a contradiction. In the case (F), considering the brideges between $\Pi\left(v_{3}\right)$ and $\Pi\left(v_{4}\right)$, we have the same contradiction. By the same argument, we can prove the nonexistence of families with monodromies $(\mathrm{J})$ or $(\mathrm{K})$ satisfying the above condition (ii).

We assume that there exists a family whose special fiber is (N) satisfying the condition (iii). Assume that $\Pi\left(v_{1}\right)=\Pi\left(v_{2}\right)$. Since $\Theta$ has no loop, we may assume that $\Pi\left(v_{3}\right)=$ $\Pi\left(v_{4}\right)$. From the condition of (iii), we know that $D_{v_{1} v_{3}} \neq D_{v_{2} v_{4}}$, although $D_{v_{1} v_{3}}$ and $D_{v_{2} v_{4}}$ are the bridges between $\Pi\left(v_{1}\right)$ and $\Pi\left(v_{3}\right)$, a contradiction to the unicity of the bridge. Thus, we may assume that $\Pi\left(v_{1}\right) \neq \Pi\left(v_{2}\right)$. Since $\Theta$ has no loop, we may assume that $\Pi\left(v_{1}\right)=\Pi\left(v_{3}\right)$ and $\Pi\left(v_{2}\right)=\Pi\left(v_{4}\right)$. In this case the double covering $\psi_{r}^{*}\left(D_{v_{1} v_{2}}\right)$ must be two distinct $\boldsymbol{P}^{1}$ chains between $v_{1}$ and $v_{2}$. On the other hand, $\psi_{r}^{*}\left(D_{v_{3} v_{4}}\right)$ must be two distinct $\boldsymbol{P}^{1}$-chains between $v_{3}$ and $v_{4}$. Since $D_{v_{1} v_{2}}=D_{v_{3} v_{4}}, \psi_{r}^{*}\left(D_{v_{1} v_{2}}\right)$ must be four distinct $\boldsymbol{P}^{1}$-chains, a contradiction to the fact that $\psi_{r}$ is a map of degree two.

Choosing a topological monodromy, we define an integer $-K_{e_{i}}$ for each edge as the sum of the valencies and the screw number at the edge ([MM1], [AI, p. 201]). These integers play a very important role when we deal with the monodromies of hyperelliptic families. In the following theorem, $c$ denotes a nonnegative integer.

THEOREM 1.8. There exist no hyperelliptic families satisfying the following conditions: (O).

(i) The topological type of the stable model is one of the types (D), (H), (I), (M) and

(ii) The topological type of the semistable model is one of the types (E), (F), (G), (J), (K), (N) satisfying the conditions (i), (ii) and (iii) in Proposition 1.7.

(iii) The monodromy satisfies one of the following:

$A_{3}$ : (i9), (i10), (i11), (i12), (i13), (i14), (i15), (i16), (i17), (i18), (i19), (i20), (i28), (i34), (i35), (i36), (i37), (i41), (i42).

$A_{2}$ : (iii2), (iii3), (iii), (iii9), (iii11), (iii13), (iii14), (iii16), (iii18), (iii28).

$A_{1}$ : (viii5), (viii 12).

$A_{0}: \quad(\mathrm{xv} 2),(\mathrm{xv} 7)$.

$B_{1 i}(i=0,1): \quad V_{1}=\{($ vii4 $),($ vii7), (vii8) $\}$.

$B_{0 i}(i=0,1): \quad V_{1}=(x i v 3)$.

$C_{111}, C_{101}, C_{001}: \quad$ Id, $V_{3}=\{($ va4 $),($ va5 $)\}$.

$C_{111}, C_{001}: \quad \mathrm{II}(1,1) V_{3}=\{(\mathrm{vb} 1),(\mathrm{vb} 2)\}$.

$E_{11}: \quad \mathrm{II}(0,1), V_{1}=\{(\mathrm{vb} 1)\}, V_{2}=\{(\mathrm{vb} 1,3,5)\}, K_{e_{1}}=2 c-1$.

$E_{11}: \quad \mathrm{II}(0,1), V_{1}=\{(\mathrm{vb} 1)\}, V_{2}=\{(\mathrm{vb} 2,4,6)\}, K_{e_{1}}=2 c$.

$E_{10}: \Pi(0,1), V_{1}=\{(\mathrm{vb} 1,3,5)\}, V_{2}=\{($ xiib1 $)\}, K_{e_{1}}=2 c-1$.

$E_{10}: \quad \amalg(0,1), V_{1}=\{(\mathrm{vb} 2,4,6)\}, V_{2}=\{($ xiib2 $)\}, K_{e_{1}}=2 c+1$.

$E_{10}: \Pi(0,1), V_{1}=\{(\mathrm{vb} 2,4,6)\}, V_{2}=\{($ xiib1 $)\}, K_{e_{1}}=2 c$.

$E_{10}: \Pi(0,1), V_{1}=\{(\mathrm{vb} 1,3,5)\}, V_{2}=\{(\mathrm{xiib} 2)\}, K_{e_{1}}=2 c$. 
$E_{00}: \quad \Pi(0,1), V_{1}=V_{2}=\{($ xiibs $)\}, K_{e_{1}}=2 c-1$.

$E_{00}: \quad \Pi(0,1), V_{1}=\left\{\left(\operatorname{xiibs}_{1}\right)\right\}, V_{2}=\left\{\left(\operatorname{xiibs}_{2}\right)\right\}, s_{1} \neq s_{2}, K_{e_{1}}=2 c$.

$F_{i j}(i, j=0,1): \quad \Pi(0,1), K_{e_{1}}=2 c-1$.

$G_{1 i}(i=0,1): \quad \mathrm{II}(0,1), V_{1}=\{(\mathrm{vb} 1,3,5)\}, K_{e_{1}}=2 c-1$.

$G_{1 i}(i=0,1): \quad \mathrm{II}(0,1), V_{1}=\{(\mathrm{vb} 2,4,6)\}, K_{e_{1}}=2 c$.

$G_{0 i}(i=0,1): \quad \mathrm{II}(0,1), V_{1}=\{(\mathrm{xiib} 1)\}, K_{e_{1}}=2 c-1$.

$G_{0 i}(i=0,1): \quad \mathrm{II}(0,1), V_{1}=\{(\mathrm{xiib} 2)\}, K_{e_{1}}=2 c$.

$J_{1}: \quad \Pi(1,4), V_{1}=\{(\mathrm{vb} 1,3,5)\}, K_{e_{1}}=2 c$.

$J_{1}: \quad \mathrm{II}(1,4), V_{1}=\{(\mathrm{vb} 2,4,6)\}, K_{e_{1}}=2 c-1$.

$J_{1}: \quad \Pi(1,6), V_{1}=\{(\mathrm{vb} 1,3,5)\}, K_{e_{1}}=2 c-1$.

$J_{1}: \quad \Pi(1,6), V_{1}=\{(\mathrm{vb} 2,4,6)\}, K_{e_{1}}=2 c$.

$J_{0}: \quad \Pi(1,4), V_{1}=\{($ xiib1 $)\}, K_{e_{1}}=2 c$.

$J_{0}: \quad \Pi(1,4), V_{1}=\{($ xiib2 $)\}, K_{e_{1}}=2 c-1$.

$J_{0}: \quad \Pi(1,6), V_{1}=\{($ xiib1 $)\}, K_{e_{1}}=2 c-1$.

$J_{0}: \quad \Pi(1,6), V_{1}=\{($ xiib2 $)\}, K_{e_{1}}=2 c$.

$K_{i}(i=0,1) \Pi(1,4), K_{e_{1}}=2 c . K_{i}(i=0,1) \Pi(1,6), K_{e_{1}}=2 c-1$.

Moreover, all monodromies listed in [AI] except those listed above can be realized as monodromies of certain hyperelliptic families.

Proof. We prove the existence of the families by giving examples of the equations in Section 2. Since we have too many cases, we write down the proof of nonexistence only for several typical cases. We call a subdivisor $Z$ of the special fiber a $\boldsymbol{P}^{1}$-chain if all the components of $Z$ are nonsingular rational curves and its dual graph is linear.

Assume that there exists a normally minimal hyperelliptic family whose topological monodromy is $A_{2}$ : (iii2). Let $X=4 C_{0}+8 C_{1}+7 C_{2}+6 C_{3}+5 C_{4}+4 C_{5}+3 C_{6}+2 C_{7}+$ $5 L_{1}+2 L_{2}+Z_{1}+\cdots+Z_{k}$ be the special fiber of the family. $C_{i}, L_{i}, Z_{i}$ are all rational curves. $C_{i}$ intersects $C_{i+1}$, and $C_{i} \cdot C_{j}=0$ if $|i-j| \geq 2$. $C_{1}$ intersects $L_{1}$, and $L_{1}$ intersects $L_{2}$. $Z_{1}+\cdots+Z_{k}$ is a $\boldsymbol{P}^{1}$-chain connecting $C_{7}$ with $L_{2}$. Since the multiplicities of $C_{1}, C_{2}$ and $L_{1}$ are distinct, $\Pi\left(C_{1}\right), \Pi\left(C_{2}\right)$ and $\Pi\left(L_{1}\right)$ are all distinct. Moreover, by Lemma 1.2, we see that $\Pi\left(C_{1}\right)$ is a component of $B_{r}$ with multiplicity four. Note that $D_{C_{1} C_{2}}+4 \Pi\left(C_{1}\right)+D_{C_{1} L_{1}}$ is the bridge $D_{C_{2} L_{1}}$ and $\tilde{\tau}\left(\psi_{r}^{*}\left(D_{C_{2} L_{1}}\right)\right)=8 C_{1}$. On the other hand, since $X-4 C_{0}-8 C_{1}-7 C_{2}-5 L_{1}$ intersects $C_{2}$ and $L_{1}$ at a point, respectively, the bridge $D_{C_{2} L_{1}}$ at least contains $\Pi\left(L_{2}\right)$. Thus, $L_{2}$ is a component of $\tilde{\tau}\left(\psi_{r}^{*}\left(D_{C_{2} L_{1}}\right)\right)$, a contradiction.

In the cases $A_{2}$ : (iii3), (iii7), (iii9), (iii1), (iii13), (iii14), (iii16), the special fibers have loops and we obtain the same contradiction if we assume the existence of hyperelliptic families with the monodromies.

We take this opportunity to point out that the picture of (iii7) in [AI, p. 217] is incorrect. The sequence $(3,2,1)$ should read $(4,3,2,1)$.

Assume that there exists a hyperelliptic family with monodromy $C_{111}(i, j=0,1)$ : Id, $V_{1}=V_{2}=\left(\right.$ iv1),$V_{3}=($ va4$)$. Let $X=3 C_{0}+2 C_{1}+2 C_{2}+2 C_{3}+C_{4}+Z_{1}+Z_{2}+L_{1}+L_{2}$ be the special fiber of the family, where $C_{i}$ are nonsingular rational curves, $L_{i}$ are elliptic curves 
and each $Z_{i}$ is the $\boldsymbol{P}^{1}$-chain that connects $C_{i}$ with $L_{i}$. Since the multiplicity of $C_{0}$ is odd, $\Pi\left(C_{0}\right)$ is not a component of $B_{r}$. Since $\Pi\left(L_{1}\right) \neq \Pi\left(L_{2}\right)$, we see that $\Pi\left(C_{i}\right)(i=0,1,2,3)$ are mutually distinct. Thus, $\Pi\left(C_{0}\right)$ intersects three distinct bridges $D_{C_{0} C_{1}}, D_{C_{0} C_{2}}$ and $D_{C_{0} C_{2}}$ at a point on $B_{r}$, respectively. It contradicts the fact that $C_{0}$ is a nonsingular rational curve. The other cases of $C_{i j 1}(i, j=0,1)$ : Id, $V_{3}=(\mathrm{va} 4,5)$ can be proved by the same argument.

Assume that there exists a hyperelliptic family with monodromy $A_{2}$ : (iii18). Let $C_{0}$ be the component with multiplicity two. The dual graph of the special fiber has a loop, and we see that $\Pi\left(C_{0}\right)$ is not a component of $B_{r}$ and has multiplicity two. Since $C_{0}$ intersects other components at six distinct points, $\Pi\left(C_{0}\right)$ intersects at least three components $D_{1}, D_{2}$ and $D_{3}$ at three distinct points. For each $D_{i}$, let $\overline{D_{i}}$ be the connected maximal subdivisor of $\Theta$ containing $D_{i}$ but not $\Pi\left(C_{0}\right)$. Each $\overline{D_{i}}$ has a component with multiplicity one, because the multiplicities of the components other than $C_{0}$ are one. It contradicts the assertion of Lemma 1.2.

The cases $A_{2}$ : (iii28), $A_{1}$ : (viii5), $A_{0}$ : (xv2), (xv7), $B_{1 i}(i=0,1): V_{1}=\{($ vii4), (vii7), (vii8) \} can be proved by the same argument.

Assume that there exists a hyperelliptic family with monodromy $B_{0 i}(i=0,1), V_{1}=$ (xiv3). Let $D=2 C_{0}+2\left(\sum Z_{j}\right)+C_{1}+C_{2}$ be the subdivisor of the special fiber $X$ as shown in [AI, p. 220, (xiv3)]. $C_{i}$ and $Z_{j}$ are nonsingular rational curves and the dual graph of $C_{0}+\sum Z_{j}$ is a loop. By the configuration of $X$, we see that $\Pi\left(C_{i}\right)$ are mutually distinct and not components of $B_{n}$. Assume that $D_{C_{0} C_{1}} \neq 0$. Since $\psi_{r}^{*}\left(D_{C_{0} C_{1}}\right)$ is contracted to a point at which $C_{0}$ intersects $C_{1}$, the multiplicity of the component $\overline{D^{\prime}}$ of $\psi_{r}^{*}\left(D_{C_{0} C_{1}}\right)$ intersecting $\tilde{\tau}^{*}\left(C_{0}\right)$ is odd. On the other hand, the component $D^{\prime}$ of $D_{C_{0} C_{1}}$ intersecting $\Pi\left(C_{0}\right)$ is a component of $B_{r}$, because $C_{0}$ intersects $C_{1}$ at a point. It contradicts the fact that the multiplicity of $\overline{D^{\prime}}=\psi_{r}^{*}\left(D^{\prime}\right)$ is odd. Thus, we see that $D_{C_{0} C_{1}}=0$, a contradiction to the configuration of the special fiber.

If there exists a hyperelliptic family $S$ whose monodromy is $A_{1}$ : (viii12), the configuration of the special fiber $X$ is as shown in Figure 1. We consider $\psi_{0}: S_{0} \rightarrow \Delta \times \boldsymbol{P}^{1}$ as in $\$ 1.1$. Let $\Gamma_{0}^{\prime}$ be the strict transform of $\Gamma_{0}$ in $W_{r}$. Since the multiplicity of each component of $X$ is greater than one, $\Gamma_{0}$ is a component of $B_{0}$. If not, the multiplicities of $\psi_{r}^{*}\left(\Gamma_{0}^{\prime}\right)$ is one, and we cannot contract $\psi_{r}^{*}\left(\Gamma_{0}^{\prime}\right)$. Assume that $\Pi\left(v_{1}\right)$ is not a component of $B_{r}$. Then, $\Pi\left(v_{1}\right)$ intersects the bridge $D_{v_{1} v_{4}}$ between $\Pi\left(v_{1}\right)$ and $\Pi\left(v_{4}\right)$ at a branch point of $\psi_{r}$. If $\Pi\left(v_{2}\right) \neq \Pi\left(v_{3}\right)$, then $\Pi\left(v_{1}\right)$ intersects the bridge between $\Pi\left(v_{1}\right)$ and $\Pi\left(v_{i}\right)(i=2,3)$ at the branch points

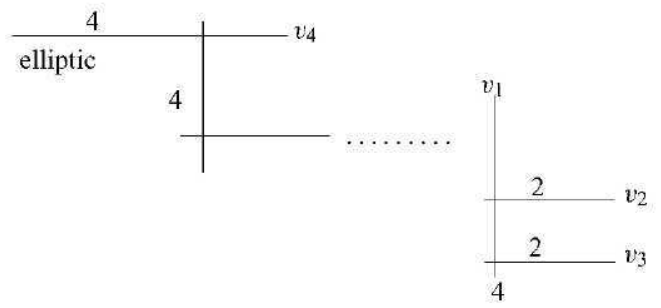

FIGURE 1. 
of $\psi_{r}$. It contradicts the fact that $v_{1}$ is a nonsingular rational curve. We may also assume that $\Pi\left(v_{2}\right)=\Pi\left(v_{3}\right)$. Since the multiplicity of $\psi_{r}^{*}\left(\Gamma_{0}^{\prime}\right)$ is equal to two, there exists a bridge $D_{v_{2} \psi_{r}^{*}\left(\Gamma_{0}^{\prime}\right)_{\mathrm{red}}}$ of $\Theta$ that does not contain $\Pi\left(v_{1}\right)$ as a component. However, the dual graph of $\psi_{r}^{*}\left(\Gamma_{0}^{\prime}+D_{v_{2} \psi_{r}^{*}\left(\Gamma_{0}^{\prime}\right)_{\text {red }}}+\Pi\left(v_{2}\right)+\Pi\left(v_{1}\right)\right)$ has a loop because $\Gamma_{0}^{\prime}$ is a component of $B_{r}$, a contradiction to the configuration of $X$. Moreover, we see that each component of $\Theta$ with multiplicity one is a component of $B_{r}$ by the same argument.

Assume that $\Pi\left(v_{1}\right)$ is a component of $B_{r}$. Then the multiplicity of $\Pi\left(v_{1}\right)$ is equal to two and $\Pi\left(v_{2}\right) \neq \Pi\left(v_{3}\right)$. We also asssume that we can find an inverse of the canonical resolution $W_{r} \stackrel{\tau_{r}}{\rightarrow} \cdots \rightarrow W_{r^{\prime}} \stackrel{\tau_{r^{\prime}}}{\rightarrow} W_{r^{\prime}-1} \rightarrow \cdots \stackrel{\tau_{1}}{\rightarrow} W_{0}$ satisfying (i) the strict transform of the exceptional set $E_{r^{\prime}}$ of $\tau_{r^{\prime}}$ by $\tau_{r^{\prime}+1} \circ \cdots \circ \tau_{r}$ is $\Pi\left(v_{1}\right)$, (ii) $\tau_{r^{\prime}}$ is the blowing-up at a point at which two components $E$ and $E^{\prime}$ of $\left(\tau_{1} \circ \cdots \circ \tau_{r^{\prime}-1}\right)^{*} \Gamma_{0}$ intersect. In this paragraph and the next, we use the same symbols $E, E^{\prime}$ and $E_{r^{\prime}}$ for the divisors on $W_{r}$ that are the strict transforms of $E$, $E^{\prime}$ and $E_{r^{\prime}}$, respectively. Since $E$ and $\Pi\left(v_{1}\right)$ are components of $B_{r}$, there exists a maximal nonzero connected subdivisor $D$ of $\Theta$ connecting $E$ with $\Pi\left(v_{1}\right)$. The component $D^{\prime}$ of $D$ intersecting $\Pi\left(v_{1}\right)$ is not a component of $B_{r}$. Moreover, since the multiplicities of $\tau_{r^{\prime}}^{*}(E)$ and $E_{r^{\prime}}$ are one and two, respectively, the multiplicity of $D^{\prime}$ is odd. Thus, the multiplicity of $\psi_{r}^{*}\left(D^{\prime}\right)$ is odd. On the other hand, the multiplicities of the components of the special fiber are all even and we cannot make a component having odd multiplicity by any sequence of the blowing-ups, a contradiction.

Thus, for each inverse of the canonical resolution, we can find the blowing-up $\tau_{r^{\prime}}$ satisfying (ii), and (iii) $\left(\tau_{r^{\prime}+1} \circ \cdots \circ \tau_{r}\right)\left(\Pi\left(v_{1}\right)\right)$ is a point on $E_{r^{\prime}}$ that is not on any other components of $\left(\tau_{1} \circ \cdots \circ \tau_{r^{\prime}}\right)^{*}\left(\Gamma_{0}\right)$. If $E_{r^{\prime}}$ is a component of $B_{r}$, we have the same contradiction as in the previous paragraph. Thus, we may assume that $E_{r^{\prime}}$ is not a component of $B_{r}$. Since the dual graph of the special fiber has no loop, we see that the divisor connecting $\Pi\left(v_{1}\right)$ with $E, E^{\prime}$ and $\Pi\left(v_{1}\right)$ intersects $E_{r^{\prime}}$ at three distinct branch points of $\psi_{r}$. It contradicts the fact that the nonrational component of the special fiber has multiplicity four.

LEMMA 1.9. The monodromy of a hyperelliptic family whose special fiber is as in Figure 2 is $E_{11}$ : $\mathrm{I}(0,1), V_{1}=(\mathrm{vb} 1), V_{2}=(\mathrm{vb} 6)$.

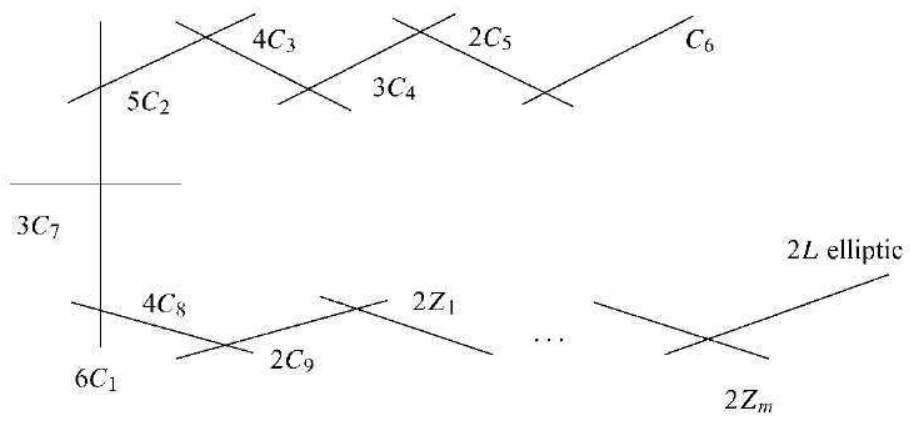

FIGURE 2. 
Since the special fiber of a hyperelliptic family with monodromy $C_{111}$ : $\Pi(1,1), V_{3}=$ (vb1), $V_{1}=V_{2}=$ (iv1) has the same configuration of the special fiber as that of a family with monodromy $E_{11}$ : II $(0,1), V_{1}=(\mathrm{vb} 1), V_{2}=(\mathrm{vb} 6)$, we obtain the following:

COROLLARY 1.10 . There exists no hyperelliptic family with monodromy $C_{111}$ : $\Pi(1,1)$, $V_{3}=(\mathrm{vb} 1), V_{1}=V_{2}=(\mathrm{iv} 1)$.

PRoOF OF Lemma 1.9. Assume that there exists a hyperelliptic family with the special fiber $X=6 C_{1}+5 C_{2}+4 C_{3}+3 C_{4}+2 C_{5}+C_{6}+3 C_{7}+4 C_{8}+2 C_{9}+2 \sum Z_{j}+2 L$ as in Figure 2. Note that $C_{i}$ and $Z_{j}$ are nonsingular rational components and $L$ is an elliptic curve. By Lemma 1.2, we see that $\Pi\left(C_{1}\right)$ is a component of $B_{r}$. Assume that the bridge $D_{C_{1} C_{2}} \neq 0$. Since $\psi_{r}^{*}\left(D_{C_{1} C_{2}}\right)$ is contracted to a point at which $C_{1}$ intersects $C_{2}$, each multiplicity of a component of $\psi_{r}^{*}\left(D_{C_{1} C_{2}}\right)$ is written as $6 a+5 b$ with positive integers $a$ and $b$. We see that the multiplicities of the components of $D_{C_{1} C_{2}}$ are at least eight, because the multiplicities of the components of $\psi_{r}^{*}\left(D_{C_{1} C_{2}}\right)$ are greater than or equal to eleven if odd, and sixteen, otherwise. Then, by any inverse of the canonical resolution, the bridge $D_{C_{1} C_{2}}$ is contracted to a point $P \in W_{r^{\prime}}$ before $\Pi\left(C_{1}\right)$ and $\Pi\left(C_{2}\right)$ are contracted. If $P$ is a bad point, $\psi_{r}^{*}\left(D_{C_{1} C_{2}}\right)$ is not contracted, because $\psi_{r}^{*}\left(D_{C_{1} C_{2}}\right)$ is the resolution graph of the singular point $\psi_{r^{\prime}}^{-1}(P)$. Thus, we see that $P$ is not a bad point, a contradiction to the process of the canonical resolution. (According to our definition of the canonical resolution, we do not blow up a non-bad point.) By the same argument, we see that $D_{C_{1} C_{7}}=D_{C_{1} C_{8}}=D_{C_{i} C_{i+1}}=0(1 \leq i \leq 5)$ and $\Pi\left(C_{8}\right)$ is not a component of $B_{r}$.

Assume that $\Pi\left(C_{9}\right)$ is not a component of $B_{r}$. Since $\Pi\left(C_{8}\right)$ also is not a component of $B_{r}$, we have $D_{C_{8} C_{9}} \neq 0$. Since the multiplicities of the components of $\psi_{r}^{*}\left(D_{C_{8} C_{9}}\right)$ are greater than or equal to six, those of the components of $D_{C_{8} C_{9}}$ are greater than or equal to three. Thus, by any inverse of the canonical resolution, $\Pi\left(C_{8}\right)+D_{C_{8} C_{9}}$ is contracted before $\Pi\left(C_{1}\right)$ and $\Pi\left(C_{9}\right)$ are contracted. Especially, $\Pi\left(C_{8}\right)$ is contracted before $\Pi\left(C_{1}\right)$ and $\Pi\left(C_{9}\right)$. Since the multiplicities of $\Pi\left(C_{1}\right)$ and $\Pi\left(C_{8}\right)$ are three and four, there should exist a component of $D_{C_{8} C_{9}}$ having multiplicity one, a contradiction. Thus, we see that $\Pi\left(C_{9}\right)$ is a component of $B_{r}$ with multiplicity one and $D_{C_{8} C_{9}}=0$.

Assume that we can find an inverse of the canonical resolution $W_{r} \stackrel{\tau_{r}}{\rightarrow} \cdots \rightarrow W_{r^{\prime}} \stackrel{\tau_{r^{\prime}}}{\rightarrow}$ $W_{r^{\prime}-1} \rightarrow \cdots \stackrel{\tau_{1}}{\rightarrow} W_{0}$ such that $\tau_{r^{\prime}}$ is a blowing-up at the point at which two components $E$ and $E^{\prime}$ of $\left(\tau_{1} \circ \cdots \circ \tau_{r^{\prime}-1}\right)^{*}\left(\Gamma_{0}\right)$ intersect and $\left(\tau_{r^{\prime}+1} \circ \cdots \circ \tau_{r^{\prime}}\right) \Pi(L)$ is a point $Q$ on $E_{r^{\prime}}$. We use the same symbols $E, E^{\prime}$ and $E_{r^{\prime}}$ for the strict transform of them on $W_{r}$. We may assume that $E$ is a component of $D_{C_{9} L}$. By the configuration of $X$, we see that $E$ and $E^{\prime}$ are components of $B_{r}$. If we assume that $E_{r^{\prime}}$ is a component of $B_{r}, \psi_{r}^{*}\left(E_{r^{\prime}}\right)$ is not contracted by $\tilde{\tau}$, because the multiplicities of $\psi_{r}^{*}\left(E_{r^{\prime}}\right)$ and $\psi_{r}^{*}\left(E^{\prime}\right)$ are four and two, respectively, a contradiction to the configuration of $X$. If we assume that $E_{r^{\prime}}$ is not a component of $B_{r}$, we see that $\psi_{r}^{*}\left(E_{r^{\prime}}\right)$ is not a nonsingular rational curve because the dual graph of $X$ has no loop and the divisor connecting $E_{r^{\prime}}$ with $E, E^{\prime}$ and $\Pi(L)$ intersects $E_{r^{\prime}}$ at three distinct branch points of $\psi_{r}$. It contradicts the assumption $\Pi(L) \neq E_{r^{\prime}}$. 


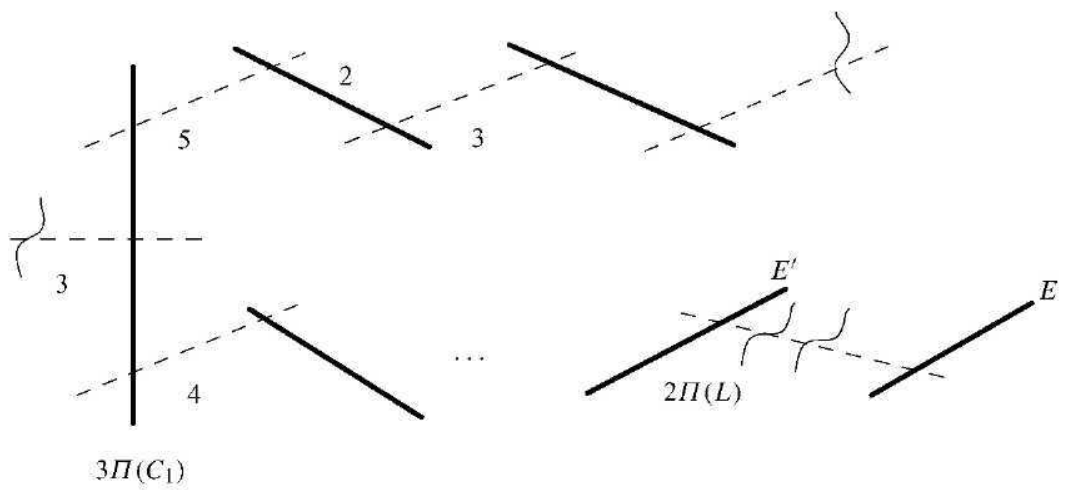

FIGURE 3 ,

$v_{1}$

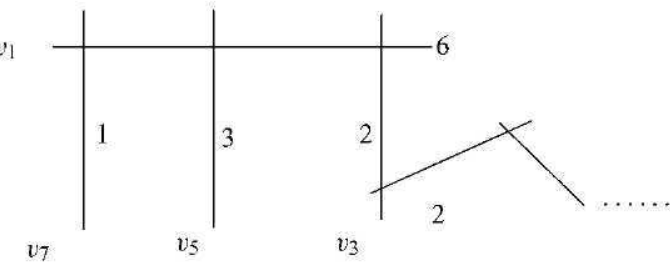

FIGURE 4 .

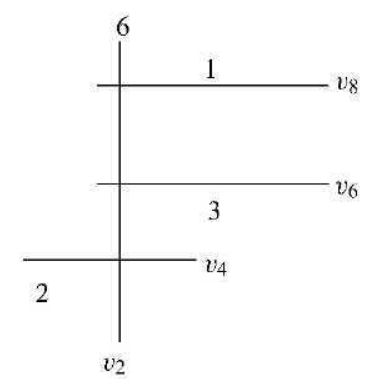

$v_{2}$

Thus, we cannot find an inverse of the canonical resolution satisfying the above condition and we obtain the figure of $\Theta$ as shown in Figure 3. In Figures 3 and 5, the dotted lines mean the components of $\Theta$ that are not the components of $B_{r}$. The solid lines mean the components of $B_{r}$, and the waves mean $\tilde{B}_{r}$. By an inverse of the canonical resolution associated to $E^{\prime}$, we obtain the equation

$$
y^{2}=t(x-1)\left\{(x-1)^{3}-t^{3 k+1}\right\}\left(x^{2}-t\right)\left(x^{2}+t\right)
$$

for a double covering of $\boldsymbol{P}^{1} \times \Delta$. By a base change of degree 6 , we see that the stable model of this family is $E_{11}$.

By the same argument, we can prove the nonexistence of hyperelliptic families with monodromies $C_{111}$ : II(1,1), $V_{3}=(\mathrm{vb} 1,2), V_{1}=V_{2}=$ (ivs) $(2 \leq s \leq 8)$.

We continue the proof of Theorem 1.8. Assume that there exists a hyperelliptic family whose monodromy is $E_{11}$ : II $(0,1), V_{1}=V_{2}=(\mathrm{vb} 2), K_{e_{1}}$ odd. The special fiber $X$ is as in Figure 4. In this case, the number of components of nonsingular rational curves between $v_{1}$ and $v_{2}$ is even. By Lemma 1.2, we know that $\Pi\left(v_{1}\right)$ and $\Pi\left(v_{2}\right)$ are components of $B_{r}$. Then, we may assume that $\Pi\left(v_{1}\right) \neq \Pi\left(v_{2}\right)$. Assume that $\Pi\left(v_{3}\right)$ is a component of $B_{r}$ with multiplicity one. Since $\psi_{r}^{*}\left(D_{v_{1} v_{3}}\right)$ is contracted to a point at which $v_{1}$ intersects $v_{3}$, 


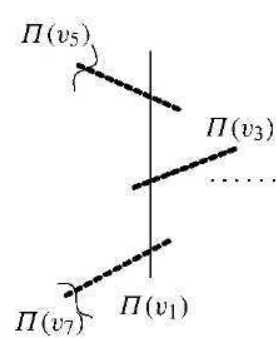

(a)

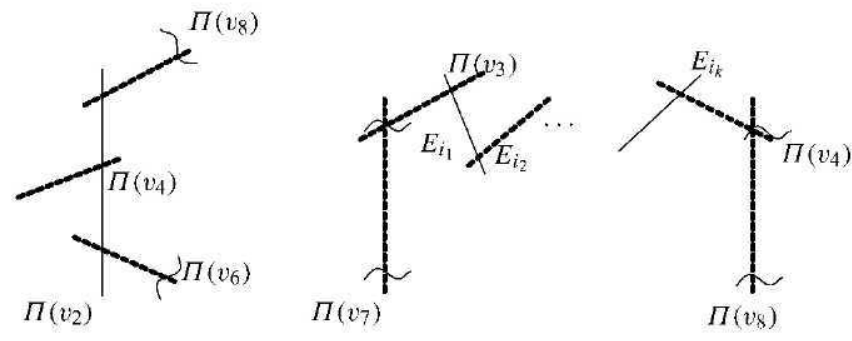

(b)

FIGURE 5

the multiplicities of the components $D_{v_{1} v_{3}}$ are greater than three. Thus, by an inverse of the canonical resolution, $\Pi\left(v_{1}\right)$ and $\Pi\left(v_{3}\right)$ cannot be contracted before $D_{v_{1} v_{3}}$ is contracted. Let $W_{r} \stackrel{\tau_{r}}{\rightarrow} \cdots \rightarrow W_{r^{\prime}} \stackrel{\tau_{r^{\prime}}}{\rightarrow} W_{r^{\prime}-1} \rightarrow \cdots \stackrel{\tau_{1}}{\rightarrow} W_{0}$ be an inverse of the canonical resolution such that the composite of the blowing-downs $\tau_{r^{\prime}} \circ \cdots \circ \tau_{r}$ contracts $D_{v_{1} v_{3}}$ but not $\Pi\left(v_{1}\right)$ and $\Pi\left(v_{3}\right)$. Let $P$ be the point at which $\left(\tau_{r^{\prime}} \circ \cdots \circ \tau_{r}\right)\left(\Pi\left(v_{1}\right)\right)$ and $\left(\tau_{r^{\prime}} \circ \cdots \circ \tau_{r}\right)\left(\Pi\left(v_{3}\right)\right)$ intersect. Since $\Pi\left(v_{1}\right)$ and $\Pi\left(v_{2}\right)$ are components of $B_{r}, \psi_{r^{\prime}-1}(P)$ is a singular point of $S_{r^{\prime}-1}$ and $\psi_{r}^{*}\left(D_{v_{1} v_{3}}\right)$ is the exceptional set of the resolution of $\psi_{r^{\prime}-1}(P)$, a contradiction to the fact that $\psi_{r}^{*}\left(D_{v_{1} v_{3}}\right)$ is contracted by $\tilde{\tau}$. Therefore, $\Pi\left(v_{3}\right)$ is not a component of $B_{r}$. By the same argument, $\Pi\left(v_{4}\right)$ also is not a component of $B_{r}$. Since we do not blow up a non-bad point as in our previous argument, we see that $\Pi\left(v_{1}\right)$ intersects $\Pi\left(v_{3}\right)$ at a point. By the same argument, $\Pi\left(v_{1}\right)$ intersects $\Pi\left(v_{l}\right)(l=5,7)$ at a point, respectively and $\Pi\left(v_{2}\right)$ intersects $\Pi\left(v_{l^{\prime}}\right)\left(l^{\prime}=4,6,8\right)$ at a point, respectively. Then the configuration of $\Theta$ is as in Figure 5, (a).

Let $\sum_{j=1}^{k} a_{i_{j}} E_{i_{j}}$ be the bridge between $v_{3}$ and $v_{4}$ such that $\Pi\left(v_{3}\right) E_{i_{1}}=1, \Pi\left(v_{4}\right) E_{i_{k}}=$ $1, E_{i_{j}} E_{i_{j+1}}=1(1 \leq j \leq k-1)$ and $E_{i_{j}} E_{i_{j^{\prime}}}=0\left(\left|j-j^{\prime}\right| \geq 2\right)$. Consider an inverse of the canonical resolution associated to $\Pi\left(v_{8}\right)$. First, we contract $\Pi\left(v_{5}\right)$ and $\Pi\left(v_{6}\right)$, then contract $\Pi\left(v_{1}\right)$ and $\Pi\left(v_{2}\right)$. Then the configuration of the image of $\Theta$ by the above contractions is as in Figure 5, (b). We use the same name for the components of $\Theta$ after contractions. Assume $a_{i_{1}} \neq 1$. Since we cannot contract $\Pi\left(v_{3}\right)$ in the next step, there exists $j^{\prime}$ such that $a_{i_{j^{\prime}}}=1$ and after some steps of blowing-downs, $E_{i_{j}}$ intersects $\Pi\left(v_{3}\right)$ at a point. If the point at which $E_{i_{j^{\prime}}}$ intersects $\Pi\left(v_{3}\right)$ is a singular point of the branch locus, $\psi_{r}^{*}\left(\sum_{j=1}^{j^{\prime}}\right)$ cannot be contracted by $\tilde{\tau}$, a contradiction to the process of the canonical resolution. Thus, $a_{i_{1}}=1$. By the same argument we see that $a_{i_{k}}=1$. If $a_{i_{2}}>2, \psi_{r}^{*}\left(E_{i_{2}}\right)$ is contracted by $\tilde{\tau}$. Let $j^{\prime}$ be the smallest integer greater than two such that $\psi_{r}^{*}\left(E_{i_{j^{\prime}}}\right)$ is not contracted by $\tilde{\tau}$. Since $a_{i_{j^{\prime}}}$ is less than or equal to two, all $E_{i_{j}}\left(1<j<j^{\prime}\right)$ are contracted to a point $P$ on $E_{i_{1}}$ before $E_{i_{1}}$ and $E_{i_{j^{\prime}}}$ by any inverse of the canonical resolution. If $P$ is a singular point of the branch locus, all $\psi_{r}^{*}\left(E_{i j}\right)\left(1<j<j^{\prime}\right)$ are not contracted, a contradiction to the process of the canonical resolution. Thus we have $a_{i_{2}}=2$ and $E_{i_{2}}$ is not a component of $B_{r}$. Repeating this argument, 
we obtain that $E_{i_{j}}$ is a component of $B_{r}$ with multiplicity one when $j$ is odd, and $E_{i_{j}}$ is not a component of $B_{r}$ and has multiplicity two, otherwise. Then the number of components of nonsingular rational curves between $v_{1}$ and $v_{2}$ is odd.

The other cases are proved by similar arguments.

2. Construction of families. In this section, we complete the proof of Theorem 1.8 by constructing hyperelliptic families whose monodromies are listed in [AI], but not listed in Theorem 1.8. More precisely, for each monodromy [ $f$ ], we give an equation for a double covering $S_{0}$ of $\boldsymbol{P}^{1} \times \Delta$ whose monodromy of the nonsingular model is $[f]$. Indices which appear in the table of symbols and equations are positive integers unless we mention their range. Let $\alpha, \alpha_{i}(1 \leq i \leq 4)$ be mutually distinct real numbers which are not integers.

Let $x$ be the inhomogeneous coordinate of $\boldsymbol{P}^{1}$ and $t$ the coordinate of $\Delta$. For example, we give an equation for $S_{0}$ whose topological monodromy is $\left(A_{3}\right)$ as follows:

$$
\left(A_{3}\right) \quad y^{2}=x(x-1)(x-2)(x-3)(x-4)(x-5)(x-6)(x-7) .
$$

We introduce some symbols for simplicity.

$$
\begin{aligned}
& F_{3}(x, t, k):=x^{3}-t^{k}, \\
& F_{12}\left(x, t, K_{1}, K_{2}\right):=\left(x-t^{K_{1}}\right)\left(x^{2}-t^{K_{2}}\right), \\
& \tilde{F}_{3}(x, t, K, L):=\left(x^{2}-t\right)^{3}-t^{K} x^{L}, \\
& \tilde{F}_{4}(x, t, K, L):=\left(x^{2}-t\right)^{4}-t^{K} x^{L}, \\
& \tilde{F}_{12}\left(x, t, K_{1}, K_{2}, L_{1}, L_{2}\right):=\left\{\left(x^{2}-t\right)-t^{K_{1}} x^{L_{1}}\right\}\left\{\left(x^{2}-t\right)^{2}-t^{K_{2}} x^{L_{2}}\right\}, \\
& \tilde{F}_{13}\left(x, t, K_{1}, L_{1}, K_{2}, L_{2}\right):=\left\{\left(x^{2}-t\right)-t^{K_{1}} x^{L_{1}}\right\}\left\{\left(x^{2}-t\right)^{3}-t^{K_{2}} x^{L_{2}}\right\}, \\
& \tilde{F}_{22}(x, t, K, L):=\left\{\left(x^{2}-t\right)^{2}-t^{K} x^{L}\right\}\left\{\left(x^{2}-t\right)^{2}+t^{K} x^{L}\right\} .
\end{aligned}
$$

Let $c$ be a positive integer. We fix a pair of integers $\left(K^{\prime}, L^{\prime}\right)$ satisfying $2 K^{\prime}+L^{\prime}-6=c$, $K^{\prime}>0$ and $0 \leq L^{\prime}<5$. We set $\tilde{F}_{3}^{c}:=\tilde{F}_{3}\left(x, t, K^{\prime}, L^{\prime}\right)$. Similarly, fixing a pair $\left(K^{\prime}, L^{\prime}\right)$ satisfying $2 K^{\prime}+L^{\prime}-8=c, K^{\prime}>0$ and $0 \leq L^{\prime}<8$, we set $\tilde{F}_{4}^{c}:=\tilde{F}_{4}\left(x, t, K^{\prime}, L^{\prime}\right)$. Fixing a pair $\left(K^{\prime}, L^{\prime}\right)$ satisfying $2 K^{\prime}+L^{\prime}-4=c$, we set $\tilde{F}_{22}^{c}:=\tilde{F}_{22}\left(x, t, K^{\prime}, L^{\prime}\right)$. Let $c_{1}$ and $c_{2}$ be positive integers. We fix two pairs of integers $\left(K_{1}^{\prime}, L_{1}^{\prime}\right)$ and $\left(K_{2}^{\prime}, L_{2}^{\prime}\right)$ satisfying $2 K_{1}^{\prime}+L_{1}^{\prime}-2=c_{1}, 2 K_{2}^{\prime}+L_{2}^{\prime}-4=c_{2}, K_{1}^{\prime}>1,0 \leq L_{1}^{\prime} \leq 1, K_{2}^{\prime}>1$ and $0 \leq$ $L_{2}^{\prime} \leq 3$. We set $\tilde{F}_{12}^{c_{1}, c_{2}}:=\tilde{F}_{12}\left(x, t, K_{1}^{\prime}, L_{1}^{\prime}, K_{2}^{\prime}, L_{2}^{\prime}\right)$. Fixing two pairs of integers $\left(K_{1}^{\prime}, L_{1}^{\prime}\right)$ and $\left(K_{2}^{\prime}, L_{2}^{\prime}\right)$ satisfying $2 K_{1}^{\prime}+L_{1}^{\prime}-2=c_{1}$ and $2 K_{2}^{\prime}+L_{2}^{\prime}-6=c_{2}$, we set $\tilde{F}_{13}^{c_{1} c_{2}}:=$ $\tilde{F}_{13}\left(x, t, K_{1}^{\prime}, L_{1}^{\prime}, K_{2}^{\prime}, L_{2}^{\prime}\right)$. We also define the following symbols using the above ones:

$$
\begin{array}{ll}
f_{1}(x, t, k, l):=x^{3}-\alpha_{1} t^{6(k-1)+3 l} . & f_{2}(x, t, k, l):=F_{3}(x, t, 6 k+3 l-1) . \\
f_{3}(x, t, k, l):=F_{3}(x, t, 6 k+3 l-5) . & f_{4}(x, t, k, l):=F_{3}(x, t, 6 k+3 l-2) . \\
f_{5}(x, t, k, l):=F_{3}(x, t, 6 k+3 l-4), & f_{6}(x, t, k, l)=F_{12}(x, t, 2 k+l, 4 k+2 l-1) . \\
f_{7}(x, t, k, l):=F_{12}(x, t, 2 k+l, 4 k+2 l-3), & f_{8}(x, t, k, l):=F_{3}(x, t, 6 k+3 l-3) . \\
g_{1}(x, t, k):=x^{5}-\alpha_{2} t^{10(k-1)} . & g_{2}(x, t, k):=x^{5}-t^{10 k-7} . \\
g_{3}(x, t, k):=x^{5}-t^{10 k-3} . & g_{4}(x, t, k):=x^{5}-t^{10 k-1} . \\
g_{5}(x, t, k):=x^{5}-t^{10 k-9} . & g_{6}(x, t, k):=x\left(x^{4}-t^{8 k-1}\right) . \\
g_{7}(x, t, k):=x\left(x^{4}-t^{8 k-3}\right) . & g_{8}(x, t, k):=x\left(x^{4}-t^{8 k-7}\right) .
\end{array}
$$




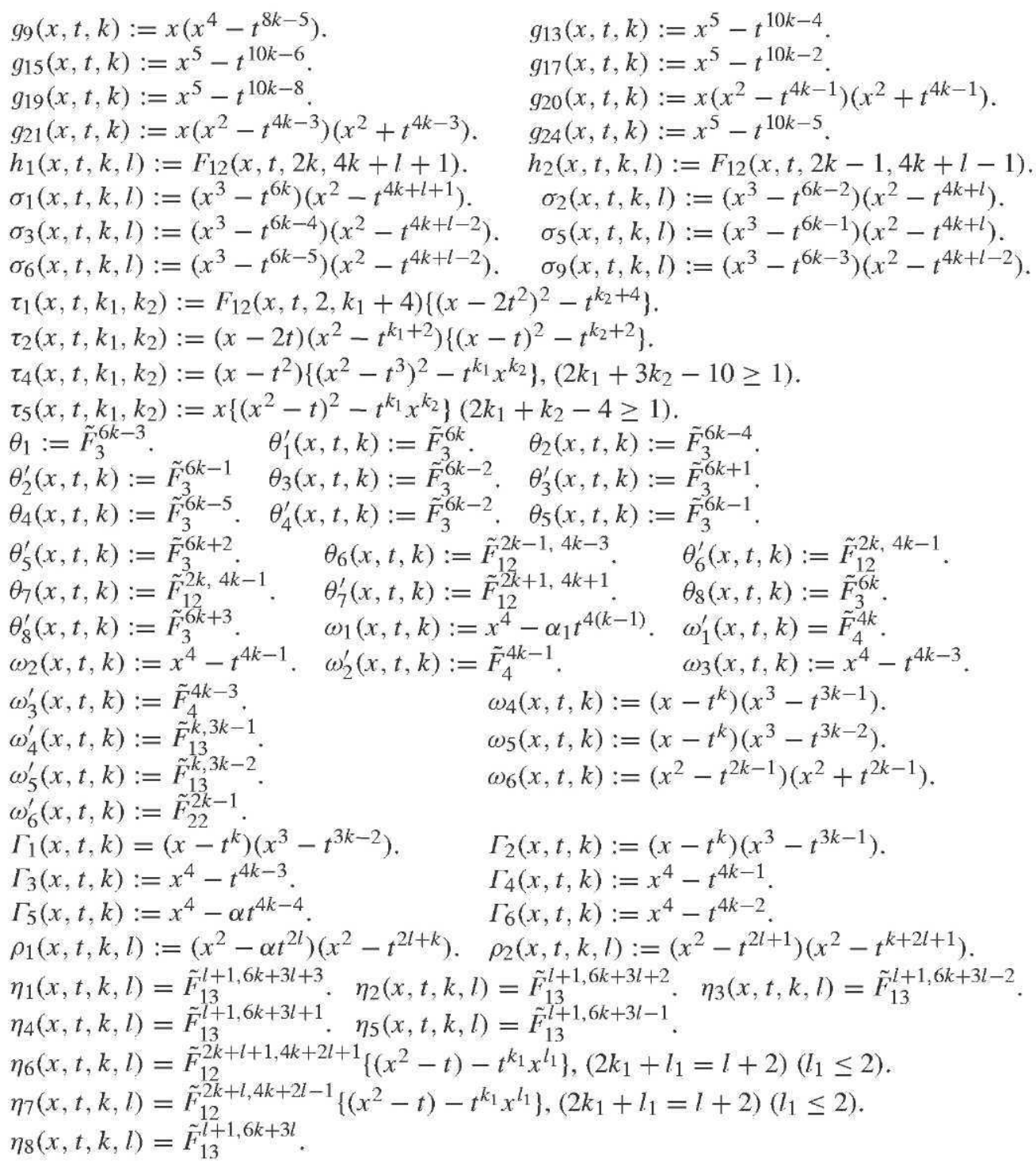

We first give examples in the semistable and periodic cases. We then give examples of hyperelliptic families whose topological monodromies are neither periodic nor semistable. In the periodic cases, it suffices to construct hyperelliptic families whose topological monodromies are (i1), (i7), (i22), (i44) and (i47). We give two or three equations for the same symbol of the topological monodromies classified in $[\mathrm{AI}]$ according to the difference of their screw numbers. In the following equations, $k, k_{i}$ are positive integers and $l, l_{i}$ are nonnegative integers unless otherwise specified. 
THE CASES OF SEMISTABLE CURVES.

$\left(A_{3}\right) \quad y^{2}=x(x-1)(x-2)(x-3)(x-4)(x-5)(x-6)(x-7)$.

$\left(A_{2}\right) \quad y^{2}=\left(x^{2}-t^{k}\right)(x-1)(x-2)(x-3)(x-4)(x-5)(x-6)$.

(A $) y^{2}=\left(x^{2}-t^{k_{1}}\right)\left\{(x-1)^{2}-t^{k_{2}}\right\}(x-2)(x-3)(x-4)(x-5)$.

$\left(A_{0}\right) \quad y^{2}=\left(x^{2}-t^{k_{1}}\right)\left\{(x-1)^{2}-t^{k_{2}}\right\}\left\{(x-2)^{2}-t^{k_{3}}\right\}(x-3)(x-4)$.

$\left(B_{21}\right) \quad y^{2}=\left(x^{3}-t^{6 k}\right)(x-1)(x-2)(x-3)(x-4)(x-5)$.

$\left(B_{20}\right) y^{2}=\left(x-t^{2 k}\right)\left(x^{2}-t^{4 k+k_{1}}\right)(x-1)(x-2)(x-3)(x-4)(x-5)$.

$\left(B_{11}\right) \quad y^{2}=\left(x^{3}-t^{6 k_{1}}\right)\left\{(x-1)^{2}+t^{k_{2}}\right\}(x-2)(x-3)(x-4)$.

$\left(B_{10}\right) \quad y^{2}=\left(x-t^{2 k_{1}}\right)\left(x^{2}-t^{4 k_{1}+k_{2}}\right)\left\{(x-1)^{2}+t^{k_{3}}\right\}(x-2)(x-3)(x-4)$.

$\left(B_{01}\right) \quad y^{2}=\left(x^{3}-t^{6 k_{1}}\right)\left\{(x-1)^{2}+t^{k_{2}}\right\}\left\{(x-2)^{2}+t^{k_{3}}\right\}(x-3)$.

$\left(B_{00}\right) \quad y^{2}=\left(x-t^{2 k_{1}}\right)\left(x^{2}-t^{4 k_{1}+k_{2}}\right)\left\{(x-1)^{2}+t^{k_{3}}\right\}\left\{(x-2)^{2}+t^{k_{4}}\right\}(x-4)$.

$\left(C_{111}\right) \quad y^{2}=\left(x^{3}-t^{6 k_{1}}\right)\left\{(x-1)^{3}-t^{6 k_{2}}\right\}(x-3)(x-4)$.

$\left(C_{110}\right) \quad y^{2}=\left(x^{3}-t^{6 k_{1}}\right)\left\{(x-1)^{2}-t^{k_{2}}\right\}\left\{(x-2)^{3}-t^{6 k_{3}}\right\}$.

$\left(C_{011}\right) \quad y^{2}=\left(x-t^{2 k_{1}}\right)\left(x^{2}-t^{4 k_{1}+k_{2}}\right)\left\{(x-1)^{3}-t^{6 k_{3}}\right\}(x-3)(x-4)$.

$\left(C_{001}\right) \quad y^{2}=\left(x+t^{2 k_{1}}\right)\left(x^{2}-t^{4 k_{1}+k_{2}}\right)\left(x-2+t^{2 k_{3}}\right)\left\{(x-2)^{2}-t^{4 k_{3}+k_{4}}\right\}(x-3)(x-4)$.

$\left(C_{010}\right) \quad y^{2}=\left(x+t^{2 k_{1}}\right)\left(x^{2}-t^{4 k_{1}+k_{2}}\right)\left(x-1+t^{2 k_{3}}\right)\left\{(x-2)^{3}-t^{6 k_{4}}\right\}$.

$\left(C_{000}\right) \quad y^{2}=\left(x+t^{2 k_{1}}\right)\left(x^{2}-t^{4 k_{1}+k_{2}}\right)\left\{(x-2)^{2}-t^{k_{3}}\right\}\left(x-3+t^{2 k_{4}}\right)\left\{(x-3)^{2}-t^{4 k_{4}+k_{5}}\right\}$.

$\left(E_{11}\right) \quad y^{2}=\left(x^{4}-t^{4 k}\right)(x-1)(x-2)(x-3)(x-4)$.

$\left(E_{01}\right) \quad y^{2}=\left(x^{2}-t^{2 k_{1}}\right)\left(x^{2}-t^{2 k_{1}+k_{2}}\right)(x-1)(x-2)(x-3)(x-4)$.

$\left(E_{00}\right) \quad y^{2}=\left(x^{2}-t^{2 k_{1}}\right)\left(x^{2}-t^{2 k_{1}+k_{2}}\right)\left\{(x-1)^{2}-t^{k_{3}}\right\}(x-3)(x-4)$.

$\left(F_{11}\right) \quad y^{2}=\left(x-t^{k_{1}}\right)\left(x^{3}-t^{3 k_{1}+6 k_{2}}\right)\left\{(x-1)^{3}-t^{6 k_{3}}\right\}(x-2)$.

$\left(F_{01}\right) \quad y^{2}=\left(x-t^{k_{1}}\right)\left(x^{3}-t^{3 k_{1}+6 k_{2}}\right)\left(x-1+t^{2 k_{3}}\right)\left\{(x-1)^{2}-t^{4 k_{3}+k_{4}}\right\}(x-2)$.

$\left(F_{00}\right) \quad y^{2}=\left(x-1+t^{2 k_{1}}\right)\left\{(x-1)^{2}-t^{4 k_{1}+k_{2}}\right\}\left(x-\alpha t^{k_{3}}\right)\left(x-t^{k_{3}+2 k_{4}}\right)\left(x^{2}-t^{2 k_{3}+4 k_{4}+k_{5}}\right)(x-2)$.

$\left(G_{11}\right) \quad y^{2}=\left(x-t^{k_{1}}\right)\left(x^{3}-t^{3 k_{1}+6 k_{2}}\right)(x-1)(x-2)(x-3)(x-4)$.

$\left(G_{10}\right) \quad y^{2}=\left(x-t^{k_{1}}\right)\left(x-t^{k_{1}+2 k_{2}}\right)\left(x^{2}-t^{2 k_{1}+4 k_{2}+k_{3}}\right)(x-1)(x-2)(x-3)(x-4)$.

$\left(G_{01}\right) \quad y^{2}=\left(x-t^{k_{1}}\right)\left(x^{3}-t^{3 k_{1}+6 k_{2}}\right)\left\{(x-1)^{2}-t^{k_{3}}\right\}(x-2)(x-3)$.

$\left(G_{00}\right) \quad y^{2}=\left(x-\alpha t^{k_{1}}\right)\left(x-t^{k_{1}+2 k_{2}}\right)\left(x^{2}-t^{2 k_{1}+4 k_{2}+k_{3}}\right)\left\{(x-1)^{2}-t^{l_{2}}\right\}(x-2)(x-3)$.

$\left(J_{1}\right) \quad y^{2}=\left(x^{4}-t^{4 k_{1}}\right)\left\{(x-1)^{2}-t^{k_{2}}\right\}\left\{(x-2)^{2}-t^{k_{3}}\right\}$.

$\left(J_{0}\right) \quad y^{2}=\left(x^{2}-t^{2 k_{1}}\right)\left(x^{2}-t^{2 k_{1}+k_{2}}\right)\left\{(x-1)^{2}-t^{k_{3}}\right\}\left\{(x-2)^{2}-t^{k_{4}}\right\}$.

$\left(K_{1}\right) \quad y^{2}=\left(x-t^{k_{3}}\right)\left(x^{3}-t^{3 k_{3}+6 k_{4}}\right)\left\{(x-1)^{2}-t^{k_{1}}\right\}\left\{(x-2)^{2}-t^{k_{2}}\right\}$.

$\left(K_{0}\right) \quad y^{2}=\left(x-t^{k_{3}}\right)\left(x-t^{k_{3}+2 k_{4}}\right)\left(x^{2}-t^{2 k_{3}+4 k_{4}+k_{5}}\right)\left\{(x-1)^{2}-t^{k_{1}}\right\}\left\{(x-2)^{2}-t^{k_{2}}\right\}$.

(L) $y^{2}=\left(x^{2}-t^{k_{1}}\right)\left\{(x-1)^{2}-t^{k_{2}}\right\}\left\{(x-2)^{2}-t^{k_{3}}\right\}\left\{(x-3)^{2}-t^{k_{4}}\right\}$.

(N) $y^{2}=\left\{\left(x-t^{k_{1}}\right)^{2}-t^{2 k_{1}+k_{2}}\right\}\left\{x^{2}-t^{2 k_{1}+k_{3}}\right\}\left\{(x-1)^{2}-t^{k_{4}}\right\}\left\{(x-2)^{2}-t^{k_{5}}\right\}$.

THE PERIODIC CASES.

(i1) $y^{2}=\left(x^{7}-t^{9}\right)(x-1)$.

(i7) $y^{2}=x\left(x^{6}-t^{7}\right)(x-1)$.

(i22) $y^{2}=x^{8}+t^{3}$.

(i44) $y^{2}=t\left\{\left(x^{4}-t\right)\left(x^{4}+t\right)\right\}$.

(i47) $y^{2}=t\left\{\left(x^{2}-\alpha_{1} t\right)\left(x^{2}-\alpha_{2} t\right)\left(x^{2}-\alpha_{3} t\right)\left(x^{2}-\alpha_{4} t\right)\right\}$.

Next, we give examples of hyperelliptic families whose monodromies are neither periodic nor semistable.

THE CASES WHERE THE STABLE MODEL IS $A_{2}$.

(iii4) $y^{2}=t\left(x^{6}-t^{5}\right)\left(x^{2}-t\right), y^{2}=\left(x^{6}-t^{5}\right)\left\{(x-1)^{2}+t^{t}\right\}$. 


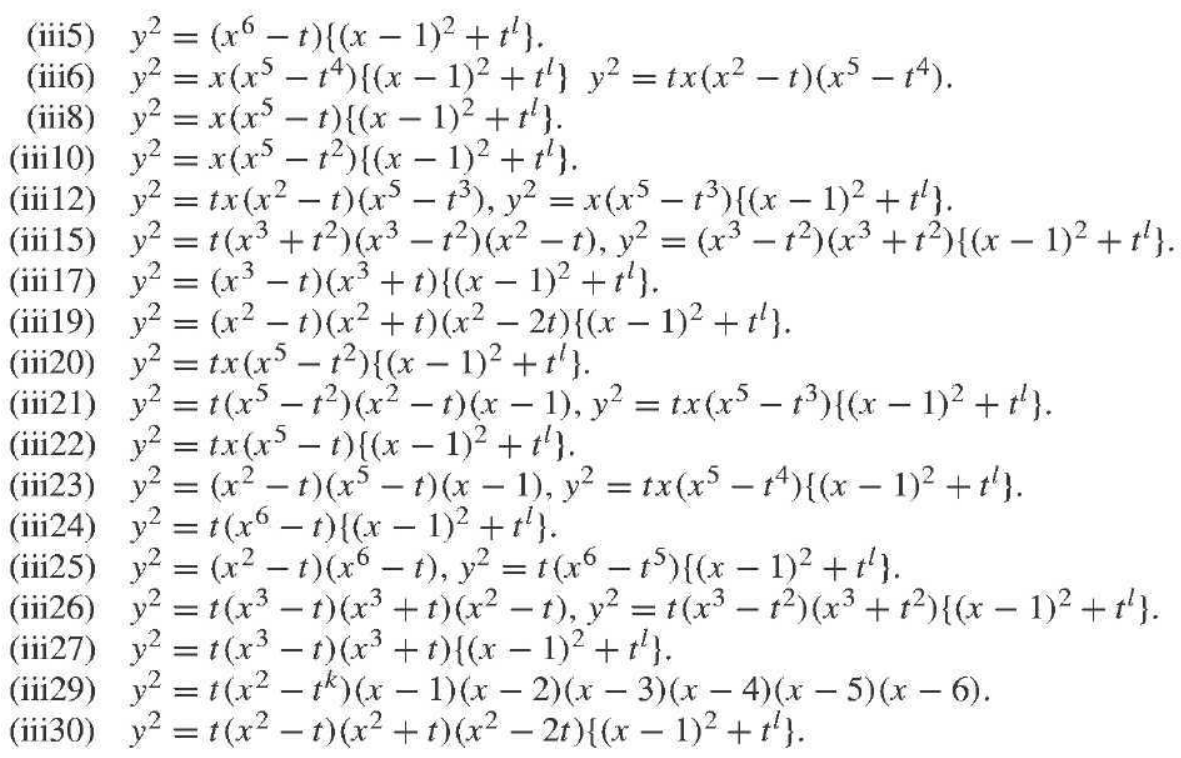

THE CASES WHERE THE STABLE MODEL IS $A_{1}$.

(viii2) $y^{2}=t\left(x^{4}-t\right)\left(x^{2}+t^{k}\right)\left\{(x-1)^{2}+t^{l}\right\}$.

(viii3) $y^{2}=t\left(x^{4}-t^{3}\right)\left(x^{2}-t^{k+1}\right)\left\{(x-1)^{2}+t^{l}\right\} . y^{2}=\left(x^{4}-t^{3}\right)\left(x^{2}-t^{k+1}\right)\left(x^{2}-t\right)$

(viii4) $y^{2}=\left(x^{2}-t^{k_{1}+1}\right)\left(x^{2}-t\right)\left(x^{2}+t\right)\left\{(x-1)^{2}-t^{k_{2}}\right\}$.

(viii6) $y^{2}=t\left(x^{2}-t^{k_{1}}\right)\left\{(x-1)^{2}-t^{k_{2}}\right\}(x-2)(x-3)(x-4)(x-5)$.

(viii7) $y^{2}=t\left(x^{2}-t^{k+1}\right)\left(x^{2}-t\right)\left(x^{2}+t\right)\left\{(x-1)^{2}+t^{l}\right\}$.

(viii8) $y^{2}=\left(x^{2}-t\right)\left(x^{2}+t\right)\left\{\left(x^{2}-2 t\right)^{2}-t^{k} x^{l}\right\},(2 k+l-2 \geq 1)$.

(viii9) $y^{2}=(x-1)\left(x^{2}-t\right)(x-t)\left\{\left(x^{2}-2 t\right)^{2}-t^{k} x^{l}\right\},(2 k+l-2 \geq 1)$.

(viii10) $y^{2}=t(x-1)\left(x^{2}-t\right)(x-t)\left\{\left(x^{2}-2 t\right)^{2}-t^{k} x^{l}\right\},(2 k+l-2 \geq 1)$.

(viii11) $y^{2}=t\left(x^{2}-t\right)\left(x^{2}+t\right)\left\{\left(x^{2}-2 t\right)^{2}-t^{k} x^{l}\right\},(2 k+l-2 \geq 1)$.

THE CASES WHERE THE STABLE MOdel IS $A_{0}$.

(xv3) $y^{2}=t\left(x^{2}-t^{k_{1}}\right)\left\{(x-1)^{2}+t^{k_{2}}\right\}\left\{(x-2)^{2}+t^{k_{3}}\right\}(x-3)(x-4)$.

(xv4) $y^{2}=t\left(x^{2}-t\right)\left\{\left(x^{2}-2 t\right)^{2}-t^{k_{1}} x^{l_{1}}\right\}\left\{(x-1)^{2}+t^{l_{2}}\right\},\left(2 k_{1}+l_{1}-2 \geq 1\right)$.

(xv5) $y^{2}=\left(x^{2}-t\right)\left\{\left(x^{2}-2 t\right)^{2}-t^{k_{1}} x^{l_{1}}\right\}\left\{(x-1)^{2}+t^{l_{2}}\right\},\left(2 k_{1}+l_{1}-2 \geq 1\right)$.

(xv6) $y^{2}=t x(x-1)\left\{\left(x^{3}-t\right)^{2}-t^{k} x^{l}\right\},(3 k+l \geq 7)$.

(xv8) $y^{2}=x(x-1)\left\{\left(x^{3}-t\right)^{2}-t^{k} x^{l}\right\},(3 k+l \geq 7)$.

THE CASES WHERE THE STABLE MODEL IS $B_{21}$.

$B_{21}: V_{1}=\left(\right.$ iis $\left.s_{1}\right), V_{2}=\left(\right.$ iv $\left.s_{2}\right) . \quad y^{2}=g_{s_{1}}\left(x, t, k_{1}\right) f_{s_{2}}\left(x-1, t, k_{2}, 0\right)$.

When $B_{21}: V_{1}=$ (iis) $V_{1}=\left(\right.$ ivs $s_{2}$ ) and the screw number is special (not appearing in the above equation), examples of their equations are as follows (we write (ii2)-(iv2) instead of writing $V_{1}=$ (ii2), $V_{2}=$ (iv2) for simplicity):

(ii2)-(iv2) $y^{2}=t\left(x^{3}-t^{2}\right)\left(x^{5}-t^{2}\right)$. (ii2)-(iv6) $y^{2}=t x\left(x^{2}-1\right)\left(x^{5}-t^{2}\right)$.

(ii3)-(iv2) $y^{2}=t\left(x^{5}-t^{2}\right)\left\{(x-1)^{3}-t^{2}\right\}$. (ii3)-(iv4) $y^{2}=t\left(x^{5}-t^{2}\right)\left\{(x-1)^{3}-t\right\}$.

(ii3)-(iv5) $y^{2}=t\left(x^{3}-t^{2}\right)\left(x^{5}-t^{3}\right)$. (ii3)-(iv6) $y^{2}=t\left(x^{5}-t^{2}\right)(x-1)\left\{(x-1)^{2}-t\right\}$. 


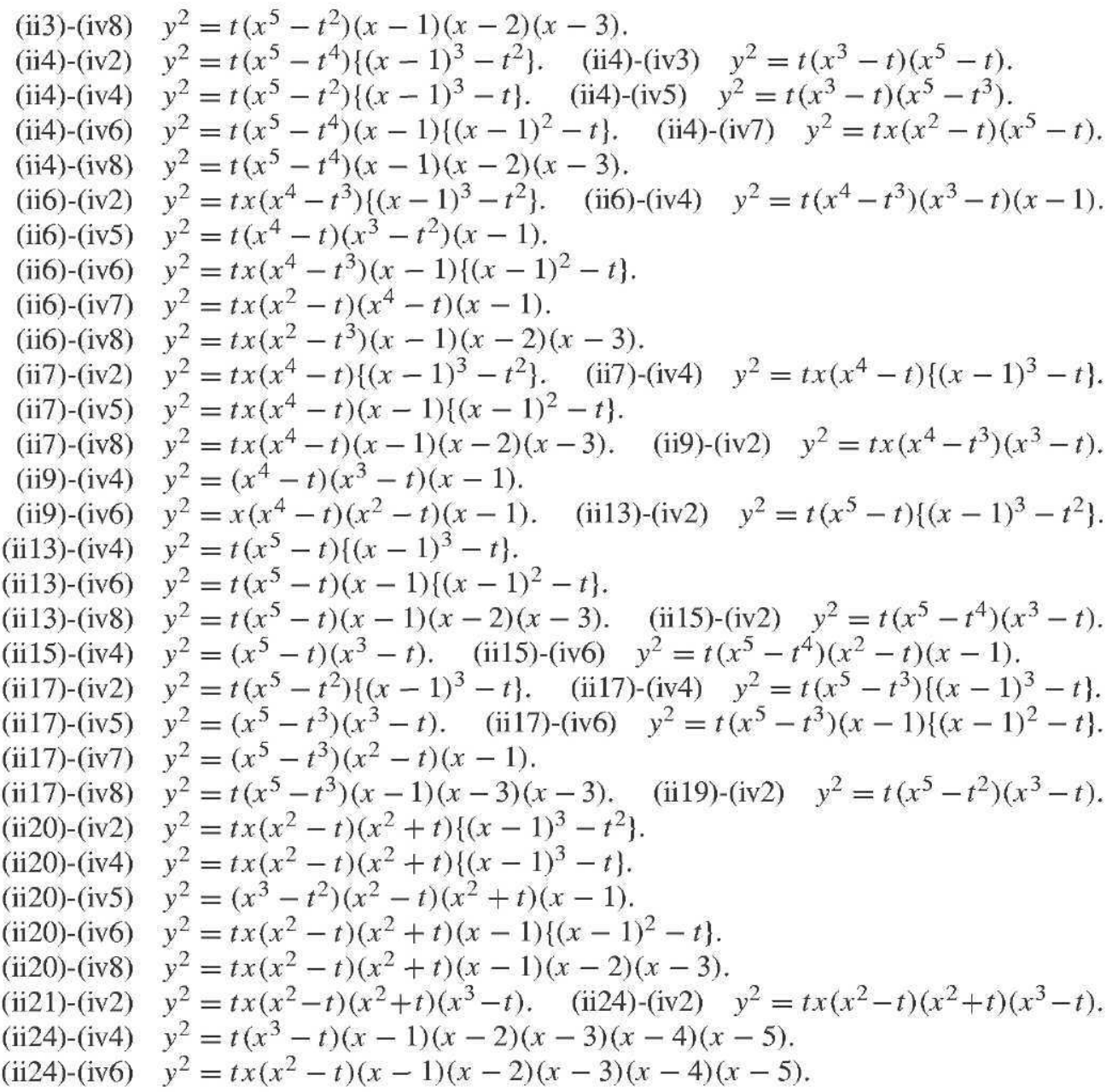

THE CASES WHERE THE STABLE MODEL IS $B_{20}$.

$B_{20}: V_{1}=\left(\right.$ iis $\left.s_{1}\right), V_{2}=\left(\right.$ xis $\left._{2}\right) . \quad y^{2}=g_{s_{1}}\left(x, t, k_{1}\right) h_{s_{2}}\left(x-1, t, k_{2}, l\right)$.

When the screw number at $e_{1}$ is special, we need the following equations in addition to those above:

$\begin{aligned} \text { (ii6)-(xi2) } & y^{2}=t x\left(x^{4}-t^{3}\right)\left\{(x-1)^{2}-t^{l}\right\}(x-2) . \\ \text { (ii13)-(xi2) } & y^{2}=t\left(x^{5}-t\right)\left\{(x-1)^{2}-t^{l}\right\}(x-2) . \\ \text { (ii17)-(xi2) } & y^{2}=t\left(x^{5}-t^{3}\right)\left\{(x-1)^{2}-t^{l}\right\}(x-2) . \\ \text { (ii20)-(xi2) } & y^{2}=t x\left(x^{2}-t\right)\left(x^{2}+t\right)\left\{(x-1)^{2}-t^{l}\right\}(x-2) .\end{aligned}$

THE CASES WHERE THE STABLE MODEL IS $B_{11}$.

$B_{11}: V_{1}=\left(\right.$ vii $\left._{1}\right), V_{2}=\left(\right.$ iv $\left.s_{2}\right) . \quad y^{2}=f_{s_{2}}(x-1, t, k, 0) \sigma_{s_{1}}\left(x, t, k_{1}, l\right)$. 
THE CASES WHERE THE STABLE MODEL IS $\boldsymbol{B}_{10}$.

$B_{10}: V_{1}=\left(\right.$ viii $\left._{1}\right), V_{2}=\left(\right.$ xis $\left._{2}\right) . \quad y^{2}=\sigma_{s_{1}}\left(x-1, t, k_{1}, l_{1}\right) h_{s_{2}}\left(x, t, k_{2}, l_{2}\right)$.

THE CASES WHERE THE STABLE MODEL IS $B_{01}$.

$B_{01}: V_{1}=\left(\operatorname{xiv} s_{1}\right), V_{2}=\left(\mathrm{iv} s_{2}\right) . \quad y^{2}=\tau_{s_{1}}\left(x, t, k_{1}, k_{2}\right) f_{s_{2}}\left(x-1, t, k_{3}, 0\right)$.

THE CASES WHERE THE STABLE MODEL IS $B_{00}$.

$B_{00}: V_{1}=\left(\operatorname{xiv} s_{1}\right), V_{2}=\left(\operatorname{xis}_{2}\right) . \quad y^{2}=\tau_{s_{1}}\left(x, t, k_{1}, k_{2}\right) h_{s_{2}}\left(x-1, t, k_{3}, l\right)$.

THE CASES WHERE THE STABLE MODEL IS $C_{111}$.

$C_{111}$ : Id, $V_{1}=\left(\mathrm{iv} s_{1}\right), V_{2}=\left(\mathrm{iv} s_{2}\right), V_{3}=$ (va1).

$$
y^{2}=f_{s_{1}}\left(x, t, k_{1}, 0\right) f_{s_{2}}\left(x-1, t, k_{2}, 0\right)(x-2)(x-3) .\left(k_{i} \geq 2 \text { when } s_{1}=1 \text { or } s_{2}=1 .\right)
$$

$C_{111}: \operatorname{Id}, V_{1}=\left(\mathrm{iv} s_{1}\right), V_{2}=\left(\mathrm{iv} s_{2}\right), V_{3}=(\mathrm{va} 2)$.

$$
y^{2}=\left(x^{2}-t^{3}\right) f_{s_{1}}\left(x, t, k_{1}, 1\right) f_{s_{2}}\left(x-1, t, k_{2}, 0\right) .\left(k_{1} \geq 0 \text { when } s_{1}=2,4,6,8 .\right)
$$

$C_{111}:$ Id, $V_{1}=\left(\mathrm{iv} s_{1}\right), V_{2}=$ (ivs $\left.s_{2}\right), V_{3}=$ (va3).

$$
y^{2}=\left(x^{2}-t\right) f_{s_{1}}\left(x, t, k_{1}, 1\right) f_{s_{2}}\left(x-1, t, k_{2}, 0\right) .
$$

$C_{111}$ : Id, $V_{1}=\left(\mathrm{iv} s_{1}\right), V_{2}=$ (ivs $\left.s_{2}\right), V_{3}=$ (va6).

$$
y^{2}=\left(x^{2}-t^{2}\right) f_{s_{1}}\left(x, t, k_{1}+1,0\right) f_{s_{2}}\left(x-1, t, k_{2}, 0\right) .
$$

$C_{111}: \mathrm{II}(1,1), V_{1}=V_{2}=$ (iv $\left.s_{1}\right), V_{3}=(\mathrm{vb} 3) . \quad y^{2}=t x(x-1) \theta_{s_{1}}(x, t, k)$.

$C_{111}$ : II $(1,1), V_{1}=V_{2}=\left(\mathrm{iv} s_{1}\right), V_{3}=$ (vb4). $\quad y^{2}=x(x-1) \theta_{s_{1}}(x, t, k)$.

$C_{111}: \mathrm{II}(1,1), V_{1}=V_{2}=$ (iv $\left.s_{1}\right), V_{3}=(\mathrm{vb} 5) . \quad y^{2}=\left(x^{2}-2 t\right) \theta_{s_{1}}^{\prime}(x, t, k)$.

$C_{111}: \mathrm{II}(1,1), V_{1}=V_{2}=\left(\mathrm{iv} s_{1}\right), V_{3}=(\mathrm{vb} 6) . \quad y^{2}=t\left(x^{2}-2 t\right) \theta_{s_{1}}^{\prime}(x, t, k)$.

THe CASES WHERE THE STABLE MODEL IS $C_{110}$.

$C_{110}$ : Id, $V_{1}=\left(\mathrm{iv} s_{1}\right), V_{2}=\left(\mathrm{iv} s_{2}\right), V_{3}=$ (xiia1).

$$
y^{2}=f_{s_{1}}\left(x, t, k_{1}, 0\right) f_{s_{2}}\left(x-2, t, k_{2}, 0\right)\left\{(x-1)^{2}-t^{k_{3}}\right\} .
$$

$C_{110}$ : Id, $V_{1}=\left(\mathrm{iv} s_{1}\right), V_{2}=\left(\mathrm{iv} s_{2}\right), V_{3}=$ (xiia2).

$$
y^{2}=t f_{s_{1}}\left(x, t, k_{1}, 1\right) f_{s_{2}}\left(x-2, t, k_{2}, 1\right)\left\{(x-1)^{2}-t^{k_{3}}\right\} .\left(k_{1} \geq 0 \text { when } s_{1}=2,4,6,8 \text {. }\right)
$$

$C_{110}$ : II $(1,1), V_{1}=V_{2}=$ (iv $\left.s_{1}\right), V_{3}=$ (xiib1). $\quad y^{2}=\left(x^{2}-t^{k_{1}+1}\right) \theta_{s_{1}}^{\prime}$.

$C_{110}$ : II $(1,1), V_{1}=V_{2}=\left(\right.$ iv $\left.s_{1}\right), V_{3}=\left(\right.$ xiib2). $\quad y^{2}=t\left(x^{2}-t^{k_{1}+1}\right) \theta_{s_{1}}^{\prime}$.

THE CASES WHERE THE STABLE MODEL IS $C_{101}$.

$C_{101}$ : Id, $V_{1}=\left(\mathrm{iv} s_{1}\right), V_{2}=\left(\mathrm{xi} s_{2}\right), V_{3}=$ (va1).

$$
y^{2}=f_{s_{1}}\left(x, t, k_{1}, 0\right) h_{s_{2}}\left(x-1, t, k_{2}, l\right)(x-2)(x-3) .
$$

$C_{101}$ : Id, $V_{1}=\left(\mathrm{iv} s_{1}\right), V_{2}=$ (xis $\left.s_{2}\right), V_{3}=$ (va2).

$$
y^{2}=\left(x^{2}-t^{3}\right) h_{s_{2}}\left(x, t, k_{2}+1 / 2, l\right) f_{s_{1}}\left(x-1, t, k_{1}, 0\right) .
$$

$C_{101}$ : Id, $V_{1}=\left(\mathrm{iv} s_{1}\right), V_{2}=\left(\mathrm{xi} s_{2}\right), V_{3}=(\mathrm{va} 3)$.

$$
y^{2}=\left(x^{2}-t\right) h_{s_{2}}\left(x, t, k_{2}+1 / 2, l\right) f_{s_{1}}\left(x-1, t, k_{1}, 0\right) .
$$

$C_{101}$ : Id, $V_{1}=\left(\mathrm{iv} s_{1}\right), V_{2}=\left(\mathrm{xi} s_{2}\right), V_{3}=$ (va6).

$$
y^{2}=\left(x^{2}-t^{2}\right) h_{s_{2}}\left(x, t, k_{2}, l\right) f_{s_{1}}\left(x-1, t, k_{1}, 0\right) .
$$

The CaSes where the STABle MODEL is $C_{100}$.

$C_{100}$ : Id $V_{1}=\left(\mathrm{iv} s_{1}\right), V_{2}=\left(\mathrm{xi} s_{2}\right), V_{3}=$ (xiial).

$$
y^{2}=f_{s_{1}}\left(x-1, t, k_{1}, 0\right) h_{s_{2}}\left(x, t, k_{2}, l\right)\left\{(x-2)^{2}-t^{k_{3}}\right\} .\left(k_{1} \geq 2 \text { when } s_{1}=1\right. \text {.) }
$$


$C_{100}$ : Id $V_{1}=\left(\right.$ ivs $\left._{1}\right), V_{2}=\left(\right.$ xis $\left._{2}\right), V_{3}=($ xiia2).

$$
y^{2}=t f_{s_{1}}\left(x-1, t, k_{1}, 1\right) h_{s_{2}}\left(x, t, k_{2}+1 / 2, l\right)\left\{(x-2)^{2}-t^{k_{3}}\right\} .
$$

THE CASES Where THE STABLE MODEL IS $\boldsymbol{C}_{001}$.

$C_{001}$ : Id, $V_{1}=\left(\right.$ xis $\left._{1}\right), V_{2}=\left(\right.$ xis $\left._{2}\right), V_{3}=($ va1 $)$.

$$
y^{2}=h_{s_{1}}\left(x-1, t, k_{1}, l\right) h_{s_{2}}\left(x, t, k_{2}, l\right)(x-2)(x-3) .
$$

$C_{001}$ : Id, $V_{1}=\left(\right.$ xis $\left._{1}\right), V_{2}=\left(\right.$ xis $\left._{2}\right), V_{3}=(\mathrm{va} 2)$.

$$
y^{2}=t\left(x^{2}-t\right) h_{s_{1}}\left(x, t, k_{1}, l_{1}\right) h_{s_{2}}\left(x-1, t, k_{2}+1 / 2, l_{2}\right) .
$$

$C_{001}$ : Id, $V_{1}=\left(\right.$ xis $\left._{1}\right), V_{2}=\left(\right.$ xis $\left._{2}\right), V_{3}=(\mathrm{va} 3)$.

$$
y^{2}=\left(x^{2}-t\right) h_{s_{1}}\left(x, t, k_{1}+1 / 2, l_{1}\right) h_{s_{2}}\left(x-1, t, k_{2}, l_{2}\right) .
$$

$C_{001}$ : Id, $V_{1}=\left(\mathrm{xi}_{1}\right), V_{2}=\left(\mathrm{xi}_{2}\right), V_{3}=(\mathrm{va} 6)$.

$$
y^{2}=\left(x^{2}-t^{2}\right) h_{s_{1}}\left(x, t, k_{1}, l\right) h_{s_{2}}\left(x-1, t, k_{2}, l\right) \text {. }
$$

$C_{001}$ : II $(1,1), V_{1}=V_{2}=(x i 1), V_{3}=(\mathrm{vb} 3) . \quad y^{2}=t x(x-1) \tilde{F}_{12}^{2 k_{1}-1,4 k_{1}+k_{2}-2}$.

$C_{001}: \mathrm{II}(1,1), V_{1}=V_{2}=(\mathrm{xi} 2), V_{3}=(\mathrm{vb} 3)$. $y^{2}=t x(x-1) \tilde{F}_{12}^{2 k_{1}, 4 k_{1}+k_{2}}$.

$C_{001}: \mathrm{II}(1,1), V_{1}=V_{2}=(\mathrm{xi} 1), V_{3}=(\mathrm{vb} 4)$. $y^{2}=x(x-1) \tilde{F}_{12}^{2 k_{1}-1,4 k_{1}+k_{2}-2}$.

$C_{001}$ : II $(1,1), V_{1}=V_{2}=(\mathrm{xi} 2), V_{3}=(\mathrm{vb} 4)$. $y^{2}=x(x-1) \tilde{F}_{12}^{2 k_{1}, 4 k_{1}+k_{2}}$.

$C_{001}$ : II $(1,1), V_{1}=V_{2}=$ (xi1), $V_{3}=$ (vb5). $y^{2}=\left\{\left(x^{2}-2 t\right)-t^{2} x\right\} \tilde{F}_{12}^{2 k_{1}, 4 k_{1}+k_{2}-2}$.

$C_{001}$ : II $(1,1), V_{1}=V_{2}=(\mathrm{xi} 2), V_{3}=(\mathrm{vb} 5)$. $y^{2}=\left\{\left(x^{2}-2 t\right)-t^{2} x\right\} \tilde{F}_{12}^{2 k_{1}-1,4 k_{1}+k_{2}-2}$.

$C_{001}$ : II $(1,1), V_{1}=V_{2}=$ (xi1), $V_{3}=(\mathrm{vb} 6)$. $y^{2}=t\left\{\left(x^{2}-2 t\right)-t^{2} x\right\} \tilde{F}_{12}^{2 k_{1}, 4 k_{1}+k_{2}}$.

$C_{001}$ : II $(1,1), V_{1}=V_{2}=(\mathrm{xi} 2), V_{3}=(\mathrm{vb} 6)$. $y^{2}=t\left\{\left(x^{2}-2 t\right)-t^{2} x\right\} \tilde{F}_{12}^{2 k_{1}-1,4 k_{1}+k_{2}-2}$.

THE CASES Where THE STABLE MODEL IS $C_{000}$.

$C_{000}$ : Id, $V_{1}=\left(x i s_{1}\right), V_{2}=\left(x i s_{2}\right), V_{3}=(x i i a 1)$.

$$
y^{2}=h_{s_{1}}\left(x, t, k_{1}, l\right) h_{s_{2}}\left(x-1, t, k_{2}, l\right)\left\{(x-2)^{2}-t^{k}\right\} \text {. }
$$

$C_{000}$ : Id, $V_{1}=\left(\mathrm{xi} s_{1}\right), V_{2}=\left(\mathrm{xi} s_{2}\right), V_{3}=(\mathrm{xiia} 2)$.

$$
y^{2}=t h_{s_{1}}\left(x, t, k_{1}+1 / 2, l_{1}\right) h_{s_{2}}\left(x-1, t, k_{2}+1 / 2, l_{2}\right)\left\{(x-3)^{2}-t^{k}\right\} .
$$

$C_{000}$ : II $(1,1), V_{1}=V_{2}=$ (xi1), $V_{3}=$ (xiib1). $\quad y^{2}=\left(x^{2}-t^{k_{1}+1}\right) \tilde{F}_{12}^{2 k_{1}, 4 k_{1}+k_{2}}$.

$C_{000}$ : II $(1,1), V_{1}=V_{2}=(\mathrm{xi} 2), V_{3}=$ (xiib1). $\quad y^{2}=\left(x^{2}-t^{k_{1}+2}\right) \tilde{F}_{12}^{2 k_{1}-1,4 k_{1}+k_{2}-2}$.

$C_{000}$ : II $(1,1), V_{1}=V_{2}=$ (xi1), $V_{3}=$ (xiib2). $\quad y^{2}=t\left(x^{2}-t^{k_{1}+1}\right) \tilde{F}_{12}^{2 k_{1}, 4 k_{1}+k_{2}}$.

$C_{000}: \mathrm{II}(1,1), V_{1}=V_{2}=(\mathrm{xi} 2), V_{3}=(\mathrm{xiib} 2) . \quad y^{2}=t\left(x^{2}-t^{k_{1}+1}\right) \tilde{F}_{12}^{2 k_{1}-1,4 k_{1}+k_{2}-2}$.

THE CASES WhERE THE STABLE MODEL IS $E_{11}$.

$E_{11}: \mathrm{Id}, V_{1}=\left(\operatorname{vas}_{1}\right), V_{2}=\left(\operatorname{vas}_{2}\right) . \quad y^{2}=\omega_{s_{1}}\left(x, t, k_{1}\right) \omega_{s_{2}}\left(x-1, t, k_{2}\right)$.

$E_{11}: \mathrm{II}(0,1), V_{1}=\left(\mathrm{vb} s_{1}\right), V_{2}=\left(\mathrm{vb}_{2}\right) . \quad y^{2}=t \Gamma_{s_{1}}\left(x, t, k_{1}\right) \Gamma_{s_{2}}\left(x-1, t, k_{2}\right)$.

We have to give more examples when the screw number at $e_{1}$ is special.

$E_{11}: \mathrm{II}(0,1), V_{1}=(\mathrm{vb} 1), V_{2}=(\mathrm{vb} 4) . \quad y^{2}=x\left(x^{4}-t\right)\left(x^{3}-t\right)$.

$E_{11}: \mathrm{II}(0,1), V_{1}=V_{2}=(\mathrm{vb} 2) . \quad y^{2}=x\left(x^{3}-t^{2}\right)\left(x^{3}-t\right)(x-1)$.

$E_{11}$ : II $(0,1), V_{1}=(\mathrm{vb} 2), V_{2}=(\mathrm{vb} 4) . \quad y^{2}=\left(x^{4}-t^{3}\right)\left(x^{3}-t\right)(x-1)$.

$E_{11}: \mathrm{II}(0,1), V_{1}=(\mathrm{vb} 2), V_{2}=(\mathrm{vb} 6) . \quad y^{2}=\left(x^{4}-t^{2}\right)\left(x^{3}-t\right)(x-1)$.

$E_{11}$ : II $(1,2), V_{1}=V_{2}=($ vas $) . \quad y^{2}=t \omega_{s}^{\prime}$. 
$E_{11}$ : II $(1,3), V_{1}=V_{2}=$ (vas). $\quad y^{2}=\omega_{s}^{\prime}$.

THE CASES WHERE THE STABLE MODELS IS $E_{10}$.

$E_{10}$ : Id, $V_{1}=\left(\operatorname{vas}_{1}\right), V_{2}=\left(\right.$ xiias $\left._{2}\right) . \quad y^{2}=\omega_{s_{1}}\left(x, t, k_{1}\right) \rho_{s_{2}}\left(x-2, t, k_{2}, l\right)$.

$E_{10}: \mathrm{II}(0,1), V_{1}=\left(\operatorname{vbs}_{1}\right), V_{2}=\left(\operatorname{xiibs}_{2}\right) . \quad y^{2}=t \Gamma_{s_{1}}\left(x, t, k_{1}\right) \rho_{s_{2}}\left(x-1, t, k_{2}, l\right)$.

We have to give more examples when the screw number at $e_{1}$ is special.

$V_{1}=(\mathrm{vb} 2), V_{2}=(\mathrm{xiib} 2) . \quad y^{2}=x\left(x^{3}-t^{2}\right)\left(x^{2}-t\right)\left\{(x-1)^{2}-t^{k-1}\right\}$.

$V_{1}=(\mathrm{vb} 4), V_{2}=$ (xiib2). $\quad y^{2}=x\left(x^{3}-t^{2}\right)\left(x^{2}-t\right)\left\{(x-1)^{2}-t^{k-1}\right\}$.

THE CASES WHERE THE STABLE MODEL IS $E_{00}$.

$E_{00}:$ Id, $V_{1}=\left(\operatorname{xiias}_{1}\right), V_{2}=\left(\right.$ xiias $\left._{2}\right) . \quad y^{2}=\rho_{s_{1}}\left(x, t, k_{1}, l_{1}\right) \rho_{s_{2}}\left(x-1, t, k_{2}, l_{2}\right)$.

$E_{00}$ : II $(0,1), V_{1}=\left(\operatorname{xiibs}_{1}\right), V_{2}=\left(\right.$ xiibs $\left._{2}\right) . \quad y^{2}=t \rho_{s_{1}}\left(x, t, k_{1}, l_{1}\right) \rho_{s_{2}}\left(x-1, t, k_{2}, l_{2}\right)$.

$E_{00}: \mathrm{II}(1,2), V_{1}=V_{2}=$ (xiial).

$y^{2}=t\left\{\left(x^{2}-t\right)^{2}-t^{k_{1}} x^{l_{1}}\right\}\left\{\left(x^{2}-t\right)^{2}-t^{k_{2}} x^{l_{2}}\right\},\left(2 k_{1}+l_{1}-4=2 k, 2 k_{2}+l_{2}-4=2 k+l\right)$.

$E_{00}: \mathrm{II}(1,2), V_{1}=V_{2}=($ xiia2).

$y^{2}=t\left\{\left(x^{2}-t\right)^{2}-t^{k_{1}} x^{l_{1}}\right\}\left\{\left(x^{2}-t\right)^{2}-t^{k_{2}} x^{l_{2}}\right\},\left(2 k_{1}+l_{1}-4=2 k_{3}-1,2 k_{2}+l_{2}-4=2 k_{3}+k_{4}\right)$.

$E_{00}: \mathrm{II}(1,3), V_{1}=V_{2}=($ xiial $)$.

$y^{2}=\left\{\left(x^{2}-t\right)^{2}-t^{k_{1}} x^{l_{1}}\right\}\left\{\left(x^{2}-t\right)^{2}-t^{k_{2}} x^{l_{2}}\right\},\left(2 k_{1}+l_{1}-4=2 k, 2 k_{2}+l_{2}-4=2 k+l\right)$.

$E_{00}: \mathrm{II}(1,3), V_{1}=V_{2}=$ (xiia2).

$y^{2}=\left\{\left(x^{2}-t\right)^{2}-t^{k_{1}} x^{l_{1}}\right\}\left\{\left(x^{2}-t\right)^{2}-t^{k_{2}} x^{l_{2}}\right\},\left(2 k_{1}+l_{1}-4=2 k_{3}-1,2 k_{2}+l_{2}-4=2 k_{3}+k_{4}\right)$.

THE CASES WHERE THE STABLE MODEL IS $F_{11}$.

$F_{11}$ : Id, $V_{1}=\left(\mathrm{iv} s_{1}\right), V_{2}=\left(\mathrm{iv} s_{2}\right)$.

$$
y^{2}=\left(x-\alpha t^{l+1}\right)(x-2) f_{s_{2}}\left(x, t, k_{1}, l+1\right) f_{s_{1}}\left(x-1, t, k_{2}, 0\right) .
$$

$\left(k_{1} \geq 2\right.$ when $s_{1}=1$ or $s_{2}=1$.)

$F_{11}: \operatorname{II}(0,1), V_{1}=\left(\mathrm{iv} s_{1}\right), V_{2}=\left(\mathrm{iv} s_{2}\right)$.

$$
y^{2}=t\left(x-\alpha t^{l+1}\right)(x-2) f_{s_{2}}\left(x, t, k_{1}, l+2\right) f_{s_{1}}\left(x-1, t, k_{2}, 0\right) \text {. }
$$

$F_{11}$ : II(2,1), $V_{1}=V_{2}=$ (ivs). $\quad y^{2}=t \eta_{s}$.

$F_{11}$ : III $(2,2), V_{1}=V_{2}=$ (ivs). $\quad y^{2}=\eta_{s}$.

THE CASES WHERE THE STABLE MODEL IS $\boldsymbol{F}_{10}$.

$F_{10}:$ Id, $V_{1}=\left(\right.$ iv $\left.s_{1}\right), V_{2}=\left(\right.$ xis $\left.s_{2}\right)$.

$$
y^{2}=\left(x-t^{l_{1}+1}\right) f_{s_{1}}\left(x, t, k_{1}, l_{1}+1\right) h_{s_{2}}\left(x-1, t, k_{2}+1, l_{2}\right)(x-2) .
$$

$F_{10}: \Pi(0,1), V_{1}=\left(\mathrm{iv} s_{1}\right), V_{2}=\left(\right.$ xis $\left._{2}\right)$.

$$
y^{2}=t\left(x-t^{t_{1}+1}\right) f_{s_{1}}\left(x, t, k_{1}, l_{1}+2\right) h_{s_{2}}\left(x-1, t, k_{2}+1 / 2, l\right)(x-2) .
$$

THE CASES WHERE THE STABLE MODEL IS $F_{00}$.

$F_{00}$ : Id, $V_{1}=\left(\right.$ xi $\left.s_{1}\right), V_{2}=\left(\right.$ xis $\left._{2}\right)$.

$$
y^{2}=\left(x-t^{l_{1}+1}\right) f_{s_{1}}\left(x, t, k_{1}+\left(l_{1}+1\right) / 2, l_{2}\right) h_{s_{2}}\left(x-1, t, k_{2}, l_{3}\right)(x-2) .
$$

$F_{00}: \Pi(0,1), V_{1}=\left(\operatorname{xis}_{1}\right), V_{2}=\left(\operatorname{xis}_{2}\right)$.

$$
y^{2}=t\left(x-t^{l_{1}+1}\right) h_{s_{1}}\left(x, t, k_{1}+\left(l_{1}+1\right) / 2, l_{2}\right) h_{s_{2}}\left(x-1, t, k_{2}+1 / 2, l_{3}\right)(x-2) .
$$

$F_{00}: \mathrm{II}(2,1), V_{1}=V_{2}=$ (xil). $\quad y^{2}=t \tilde{F}_{12}^{2 k_{2}+k_{1}, 4 k_{2}+2 k_{1}+k_{3}}\left(x^{2}-t-t^{k_{4}} x^{l}\right),\left(2 k_{4}+l-2=k_{1}\right)$.

$F_{00}: \operatorname{II}(2,1), V_{1}=V_{2}=(x i 2)$.

$$
y^{2}=t \tilde{F}_{12}^{2 k_{2}+k_{1}-1,4 k_{2}+2 k_{1}+k_{3}-2}\left(x^{2}-t-t^{k_{4}} x^{l}\right),\left(2 k_{4}+l-2=k_{1}\right) \text {. }
$$


$F_{00}: \Pi(2,2), V_{1}=V_{2}=\left(\right.$ xi1). $\quad y^{2}=\tilde{F}_{12}^{2 k_{2}+k_{1}, 4 k_{2}+2 k_{1}+k_{3}}\left(x^{2}-t-t^{k_{4}} x^{l}\right),\left(2 k_{4}+l-2=k_{1}\right)$.

$F_{00}: \Pi(2,2), V_{1}=V_{2}=(\mathrm{xi} 2)$.

$$
y^{2}=\tilde{F}_{12}^{2 k_{2}+k_{1}-1,4 k_{2}+2 k_{1}+k_{3}-2}\left(x^{2}-t-t^{k_{4}} x^{l}\right),\left(2 k_{4}+l-2=k_{1}\right) .
$$

THE CASES WHERE THE STABLE MODEL IS $G_{11}$.

$G_{11}:$ Id, $V_{1}=\left(\operatorname{vas}_{1}\right), V_{3}=\left(\right.$ ivs $\left._{2}\right)$.

$$
y^{2}=f_{s_{2}}\left(x, t, k_{1}, 0\right)(x-1) \omega_{s_{1}}\left(x-2, t, k_{2}\right) .\left(k_{2} \geq 2 \text { when } s_{2}=1 .\right)
$$

$G_{11}: \Pi(0,1), V_{1}=\left(\mathrm{vbs}_{1}\right), V_{3}=\left(\mathrm{ivs} s_{2}\right)$.

$$
y^{2}=t \Gamma_{s_{1}}\left(x, t, k_{1}\right)(x-1) f_{s_{2}}\left(x, t, k_{2}, 1\right) .\left(k_{2} \geq 0 \text { when } s_{2}=2,4,6,8 .\right)
$$

THE CASES WHERE THE STABLE MODEL IS $G_{10}$.

$G_{10}: \mathrm{Id}, V_{1}=\left(\operatorname{vas}_{1}\right), V_{3}=\left(\operatorname{xis}_{2}\right) . \quad y^{2}=\omega_{s_{1}}\left(x, t, k_{1}\right)(x-1) h_{s_{2}}\left(x-2, t, k_{2}, l\right)$.

$G_{10}: \Pi(0,1), V_{1}=\left(\operatorname{vbs} s_{1}\right), V_{3}=\left(\mathrm{xi}_{2}\right) . \quad y^{2}=t \Gamma_{s_{1}}\left(x, t, k_{1}\right)(x-1) h_{s_{2}}\left(x-2, t, k_{2}+1 / 2, l\right)$.

THE CASES OF THE STABLE MODELS ARE $G_{00}$.

$G_{00}: \mathrm{Id}, V_{1}=\left(\operatorname{xiias}_{1}\right), V_{3}=\left(\mathrm{xi}_{2}\right) . \quad y^{2}=\rho_{s_{1}}\left(x, t, k_{1}, l_{1}+1\right)(x-1) h_{s_{2}}\left(x-2, t, k_{2}, l_{2}\right)$.

$G_{00}: \Pi(0,1), V_{1}=\left(\operatorname{xiibs}_{1}\right), V_{3}=\left(\operatorname{xi}_{2}\right)$.

$$
y^{2}=t \rho_{s_{1}}\left(x, t, k_{1}, l_{1}+1\right)(x-1) h_{s_{2}}\left(x-2, t, k_{2}+1 / 2, l_{2}\right) .
$$

THE CASES OF THE STABLE MOdels ARE $G_{01}$.

$G_{01}:$ Id, $V_{1}=\left(\right.$ xiia $\left._{1}\right), V_{3}=\left(\right.$ iv $\left.s_{2}\right) . \quad y^{2}=\rho_{s_{1}}\left(x, t, k_{1}, l+1\right)(x-1) f_{s_{2}}\left(x-2, t, k_{2}, 0\right)$.

$G_{01}$ : II $(0,1), V_{1}=\left(x \operatorname{xib} s_{1}\right), V_{3}=\left(\right.$ ivs $\left.s_{2}\right) . \quad y^{2}=t \rho_{s_{1}}\left(x, t, k_{1}, l+1\right)(x-1) f_{s_{2}}\left(x-2, t, k_{2}, 1\right)$

THE CASES WHERE THE STABLE MODEL IS $J_{1}$.

$J_{1}: \mathrm{Id}, V_{1}=$ (vas).

$$
y^{2}=\omega_{s}\left(x, t, k_{1}\right)\left\{(x-1)^{2}-t^{k_{2}}\right\}\left\{(x-2)^{2}-t^{k_{3}}\right\} .\left(k_{1} \geq 2 \text { when } s=1 .\right)
$$

$J_{1}: \mathrm{II}(0,1), V_{1}=($ vas $)$.

$$
y^{2}=\omega_{s}\left(x, t, k_{1}\right)\left\{\left(x^{2}-t\right)^{2}-t^{k_{2}} x^{l}\right\},\left(2 k_{2}+l-4 \geq 1\right) .\left(k_{1} \geq 2 \text { when } s=1 .\right)
$$

$J_{1}: \operatorname{II}(1,4), V_{1}=(\mathrm{vbs}) . \quad y^{2}=t \Gamma_{s}\left(x-1, t, k_{1}\right)\left\{\left(x^{2}-t\right)^{2}-t^{k_{2}} x^{l}\right\},\left(2 k_{2}+l-4 \geq 1\right)$.

$J_{1}: \mathrm{II}(1,6), V_{1}=(\mathrm{vbs}) . \quad y^{2}=t \Gamma_{s}\left(x, t, k_{1}\right)\left\{(x-1)^{2}-t^{k_{2}}\right\}\left\{(x-2)^{2}-t^{k_{3}}\right\}$.

THE CASES WHERE THE STABLE MODELS IS $J_{0}$.

$J_{0}$ : Id, $V_{1}=$ (xiias). $\quad y^{2}=\rho_{s}\left(x, t, k_{1}, l+1\right)\left\{(x-1)^{2}-t^{k_{2}}\right\}\left\{(x-2)^{2}-t^{k_{3}}\right\}$.

$J_{0}: \mathrm{II}(0,1), V_{1}=$ (xiias). $\quad y^{2}=\rho_{s}\left(x, t, k_{1}, l_{1}+1\right)\left\{\left(x^{2}-t\right)^{2}-t^{k_{2}} x^{l}\right\},\left(2 k_{2}+l-4 \geq 1\right)$.

$J_{0}$ : II(1,4), $V_{1}=$ (xiibs). $\quad y^{2}=t \rho_{s}\left(x-1, t, k_{1}, l_{1}\right)\left\{\left(x^{2}-t\right)^{2}-t^{k_{2}} x^{l}\right\},\left(2 k_{2}+l-4 \geq 1\right)$.

$J_{0}: \mathrm{II}(1,6), V_{1}=$ (xiibs). $\quad y^{2}=t \rho_{s}\left(x, t, k_{1}, l_{1}\right)\left\{(x-1)^{2}-t^{k_{2}}\right\}\left\{(x-2)^{2}-t^{k_{3}}\right\}$.

THE CASES WHERE THE STABLE MODEL IS $K_{1}$.

$K_{1}$ : Id, $V_{4}=$ (ivs). $\quad y^{2}=\left(x-t^{l_{1}+1}\right) f_{s}\left(x, t, k_{1}+1, l_{1}+1\right)\left\{(x-1)^{2}-t^{k_{2}}\right\}\left\{(x-2)^{2}-t^{k_{3}}\right\}$.

$K_{1}: \operatorname{II}(0,1), V_{4}=(\mathrm{iv} s)$.

$y^{2}=\left\{\left(x^{2}-t\right)^{2}-t^{k_{1}} x^{l_{1}}\right\}\left\{(x-1)-t^{l_{2}+1}\right\} f_{s}\left(x-1, t, k_{2}+1, l_{2}+1\right),\left(2 k_{1}+l_{1}-4 \geq 1\right)$.

$K_{1}: \operatorname{II}(1,4), V_{4}=(\mathrm{ivs})$.

$$
y^{2}=t\left\{\left(x^{2}-t\right)^{2}-t^{k_{1}} x^{l_{1}}\right\}\left\{(x-1)-t^{l_{2}+1}\right\} f_{S}\left(x-1, t, k_{2}, l_{2}+2\right),\left(2 k_{1}+l_{1}-4 \geq 1\right) .
$$

$K_{1}: \operatorname{II}(1,6), V_{4}=(\mathrm{iv} s)$.

$$
y^{2}=t\left(x^{2}-t^{k_{1}}\right)\left\{(x-1)^{2}-t^{k_{2}}\right\}\left\{(x-2)-t^{l_{1}+1}\right\} f_{s}\left(x-3, t, k_{3}, l_{1}+2\right) .
$$


THE CASES WHERE THE STABLE MODEL IS $K_{0}$.

$K_{0}$ : Id, $V_{4}=(x i s) . \quad y^{2}=\left(x-t^{k_{1}}\right) h_{s}\left(x, t, k_{1} / 2+k_{2}, l\right)\left\{(x-1)^{2}-t^{k_{3}}\right\}\left\{(x-2)^{2}-t^{k_{4}}\right\}$.

$K_{0}: \Pi(0,1), V_{4}=$ (xis).

$$
y^{2}=\left\{\left(x^{2}-t\right)^{2}-t^{k_{1}} x^{l_{1}}\right\}\left\{(x-1)-t^{k_{2}}\right\} h_{s}\left(x-1, t, k_{2} / 2+k_{3}, l\right)\left(2 k_{1}+l_{1}-4 \geq 1\right) .
$$

$K_{0}: \Pi(1,4), V_{4}=$ (ivs).

$$
y^{2}=t\left\{\left(x^{2}-t\right)^{2}-t^{k_{1}} x^{l_{1}}\right\}\left\{(x-1)-t^{k_{2}}\right\} h_{s}\left(x-1, t, k_{3}+\left(k_{2}+1\right) / 2, l_{2}\right)\left(2 k_{1}+l_{1}-4 \geq 1\right) .
$$

$K_{0}: \Pi(1,6), V_{4}=$ (ivs).

$$
y^{2}=t\left(x^{2}-t^{k_{1}}\right)\left\{(x-1)^{2}-t^{k_{2}}\right\}\left\{(x-2)-t^{k_{3}}\right\} h_{s}\left(x-3, t, k_{4}+\left(k_{3}+1\right) / 2, l\right) .
$$

THE CASES WHERE THE STABLE MODEL IS $L$.

$L: \Pi(0,1), V_{1}=V_{2}=(\mathrm{xb})$.

$$
\left.y^{2}=\left(x^{2}-t^{k_{1}+1}\right)\left\{\left(x^{2}-t\right)^{2}-t^{k_{2}} x^{l_{1}}\right\}\left\{(x-1)^{2}+t^{l_{2}}\right)\right\},\left(2 k_{2}+l-4 \geq 1\right) .
$$

$L: \Pi(0,2), V_{1}=V_{2}=(\mathrm{xc})$.

$$
y^{2}=\left\{\left(x^{2}-t\right)^{2}-t^{k_{1}} x^{l_{1}}\right\}\left\{\left(x^{2}-t\right)^{2}-2 t^{k_{2}} x^{l_{2}}\right\} .\left(2 k_{1}+l_{1}-4 \geq 1,2 k_{2}+l_{2}-4 \geq 1\right)
$$

$L: \mathrm{II}(1,5) . \quad y^{2}=t\left\{\left(x^{2}-t\right)^{2}-t^{k_{1}} x^{l_{1}}\right\}\left\{\left(x^{2}-2 t\right)^{2}-t^{k_{2}} x^{l_{2}}\right\},\left(2 k_{1}+l_{1}-4 \geq 1,2 k_{2}+l_{2}-4 \geq 1\right)$.

$L: \Pi(1,7) . \quad y^{2}=t\left\{\left(x^{2}-t\right)^{2}-t^{k_{1}} x^{l_{1}}\right\}\left\{x^{2}-t^{l_{2}}\right\}\left\{(x-1)^{2}+t^{l_{3}}\right\},\left(2 k_{1}+l_{1}-4 \geq 1\right)$.

$L: \operatorname{II}(1,8) . \quad y^{2}=t\left(x^{2}-t^{k_{1}}\right)\left\{(x-1)^{2}-t^{k_{2}}\right\}\left\{(x-2)^{2}-t^{k_{3}}\right\}\left\{(x-3)^{2}-t^{k_{4}}\right\}$.

$L: \operatorname{III}(0,1), V_{1}=(\mathrm{vd} s) \quad y^{2}=\left\{\left(x^{3}-t\right)^{2}-t^{k_{1}} x^{l_{1}}\right\}\left\{(x-1)^{2}-t^{l}\right\},\left(3 k_{1}+l_{1}-6 \geq 1\right)$.

$$
y^{2}=\left\{\left(x^{3}-t\right)^{2}-t^{k_{1}} x^{l_{1}}\right\}\left(x^{2}-t^{k_{2}+1}\right),\left(3 k_{1}+l_{1}-6 \geq 1\right) .
$$

$L: \operatorname{IV}(0,1), V_{1}=$ (ve). $\quad y^{2}=\left\{\left(x^{4}-t\right)^{2}-t^{k_{1}} x^{l_{1}}\right\},\left(4 k_{1}+l_{1}-8 \geq 1\right)$.

$L: \operatorname{IV}(1,1), V_{1}=$ (ve). $\quad y^{2}=t\left\{\left(x^{4}-t\right)^{2}-t^{k_{1}} x^{l_{1}}\right\},\left(4 k_{1}+l_{1}-8 \geq 1\right)$.

$L: \mathrm{VI}(1,1), V_{1}=(\mathrm{vd} s) . \quad y^{2}=t\left\{\left(x^{3}-t\right)^{2}-t^{k_{1}} x^{l_{1}}\right\}\left\{(x-1)^{2}-t^{k_{2}-1}\right\},\left(3 k_{1}+2 l_{1}-6 \geq 0\right)$.

THE CASES WHERE THE STABLE MODEL IS $N$.

$N: \amalg(0,1)$.

$y^{2}=\left\{\left(x^{2}-t\right)^{2}-t^{k_{1}} x^{l_{1}}\right\}\left\{(x-1)^{2}-t^{k_{2}+2 k_{3}}\right\}\left\{\left(x-1-t^{k_{3}}\right)^{2}-t^{k_{4}+2 k_{3}}\right\},\left(2 k_{1}+l_{1}-4 \geq 1\right)$.

$N: \operatorname{II}(0,2) . \quad y^{2}=\left\{\left(x^{2}-t^{2 k_{1}-1}\right)^{2}-t^{k_{2}+4 k_{1}-4} x^{l_{1}}\right\}\left\{\left(\left(x^{2}-1\right)^{2}-t\right)^{2}-t^{k_{3}} x^{l_{2}}\right\}$,

$$
\left(2 k_{2}+l_{1}-4 \geq 1,2 k_{3}+l_{2}-4 \geq 1\right) \text {. }
$$

$N: \mathrm{II}(2,3) . \quad y^{2}=t\left\{\left(x^{2}-t^{2 k_{1}-1}\right)^{2}-t^{k_{2}+4 k_{1}-4} x^{l_{1}}\right\}\left\{\left(\left(x^{2}-1\right)^{2}-t\right)^{2}-t^{k_{3}} x^{l_{2}}\right\}$,

$$
\left(2 k_{2}+l_{1}-4 \geq 1,2 k_{3}+l_{2}-4 \geq 1\right) \text {. }
$$

$N: \mathrm{II}(2,4) . \quad y^{2}=t\left\{\left(x-t^{k_{1}}\right)^{2}-t^{2 k_{1}+k_{2}}\right\}\left\{x^{2}-t^{2 k_{1}+k_{3}}\right\}\left\{(x-1)^{2}-t^{k_{4}}\right\}\left\{(x-2)^{2}-t^{k_{5}}\right\}$.

$N: \mathrm{II}(2,5)$.

$y^{2}=t\left\{(x-1)^{2}-t^{k_{1}}\right\}\left\{(x-2)^{2}-t^{k_{2}}\right\}\left\{\left(x^{2}-t^{2 k_{3}-1}\right)^{2}-t^{4 k_{3}+k_{4}-4} x^{l_{2}}\right\},\left(2 k_{4}+l_{2}-4 \geq 1\right)$. $N: \mathrm{II}(2,7) . \quad y^{2}=t\left\{\left(\left(x^{2}-t\right)-t^{k_{1}} x^{l_{1}}\right)^{2}-t^{k_{2}} x^{l_{2}}\right\}\left\{\left(x^{2}-t\right)^{2}-t^{k_{3}} x^{l_{3}}\right\}$,

$N: \mathrm{II}(2,8) . \quad y^{2}=\left\{\left(\left(x^{2}-t\right)-t^{k_{1}} x^{l_{1}}\right)^{2}-t^{k_{2}} x^{l_{2}}\right\}\left\{\left(x^{2}-t\right)^{2}-t^{k_{3}} x^{l_{3}}\right\}$,

$$
\left(2 k_{3}-4 k_{1}+l_{3}-2 l_{1} \geq 0,2 k_{2}+l_{2}-4 k_{1}-2 l_{1} \geq 1\right) \text {. }
$$

$$
\left(2 k_{3}-4 k_{1}+l_{3}-2 l_{1} \geq 0,2 k_{2}+l_{2}-4 k_{1}-2 l_{1} \geq 1\right) \text {. }
$$

$N: \operatorname{IV}(2,1) . \quad y^{2}=t\left\{\left(x^{2}-t\right)^{2}-t^{k_{1}} x\right\}^{2}-t^{k_{2}} x^{l},\left(2 k_{1}-3 \geq 0,2 k_{2}+l-8 \geq 0\right)$

$N$ : IV(2,2). $\quad y^{2}=\left\{\left(x^{2}-t\right)^{2}-t^{k_{1}} x\right\}^{2}-t^{k_{2}} x^{l},\left(2 k_{1}-3 \geq 0,2 k_{2}+l-8 \geq 0\right)$. 


\section{REFERENCES}

[AMO] M. Asada, M. Matsumoto And TAKayuki OdA, Local monodromy on the fundamental groups of algebraic curves along a degenerate stable curves, J. Pure Appl. Algebra 103 (1995), 235-283.

[AI] T. ASHIKAGA AND M. IsHIZAKA, Classification of degenerations of curves of genus three via MatsumotoMontesinos' theorem, Tohoku Math. J. 54 (2002), 195-226.

[DM] P. Deligne And D. MumFord, The irreducibility of the space of curves of given genus, Inst. Hautes Études Sci. Publ. Math. 36 (1969), 75-109.

[ES] C. J. EARLE AND P. L. SIPE, Families of Riemann surfaces over the punctured disk, Pacific J. Math. 150 (1991), 79-86.

[F] C. FABER, Chow rings of moduli spaces of curves I: The Chow ring of $\bar{M}_{3}$, Ann. of Math. 132 (1990), 331-419.

[Ha] J. HARveY, Cyclic groups of automorphisms of compact Riemann surfaces, Quart. J. Math. Oxford Ser. (2) 17 (1966), 86-97.

[HM] J. HARRIS AND I. MorRison, Moduli of curves, Springer-verlag, New York, 1998.

[Ho1] E. HoRIKAWA, On deformations of quintic surfaces, Invent. Math. 31 (1975), 43-85.

[Ho2] E. HORIKAWA, On algebraic surfaces with pencils of curves of genus 2, in Complex Analysis and Algebraic Geometry, a collection of papers dedicated to K. Kodaira, 79-90, Iwanami Shoten, Tokyo and Cambridge Univ. Press, 1977.

[Im] Y. ImAYoshI, Holomorphic families of Riemann surfaces and Teichm̈uller spaces, in Riemann Surfaces and Related Topics, Ann. of Math. Stud. 97 (1981), 277-300.

[Ke] S. P. KERCHHOFF, The Nielsen realization problem, Ann. of Math. 117 (1983), 235-265.

[MM1] Y. Matsumoto and J. M. Montesinos-AmilibIA, Pseudo-periodic maps and degeneration of Riemann surfaces I, II, preprints, Univ. of Tokyo and Univ. Complutense de Madrid, 1991/1992.

[MM2] Y. Matsumoto and J. M. Montesinos-Amilibia, Pseudo-periodic homeomorphisms and degeneration of Riemann surfaces, Bull. Amer. Math. Soc. 30 (1994), 70-75.

[Ni1] J. NIELSEN, Die Struktur periodischer Transformationen von Flächen, Mat.-Fys. Medd. Danske Vid. Selsk. 15 (1937), English translation: in Collected Papers 2, Birkhäuser, 1986.

[Ni2] J. NiELSEN, Surface transformation classes of algebraically finite type, Mat.-Fys. Medd. Danske Vid. Selsk. 21 (1944), 3-89.

[ST] H. SHIGA AND H. TANIGAWA, On the Maskit coordinates of Teichmüller spaces and modular transformation, Kodai Math. J. 12 (1989), 437-443.

[Wim] A. WIMAN, Über die hyperelliptischen Kurven und diejenigen vom Geschlechte=3, welche eindeutige Transformationen in sich zulassen, Bihang. Kengle. Svenska Vetenkaps-Akademiens Hendlingar, Stockholm, 1895-6.

Mathematical InSTITUTE

TOHOKU UNIVERSITY

SENDAI 980-8578

JAPAN 